LARGE-SCALE BIOLOGY ARTICLE

\title{
2 Accurate transcription start sites enable mining for the cis- 3 regulatory determinants of tissue specific gene expression
}

Mitra Ansariola ${ }^{1,3 \dagger}$, Valerie N. Fraser ${ }^{1,3 \dagger}$, Sergei A. Filichkin ${ }^{3,4}$, Maria G. Ivanchenko ${ }^{3,4}$, Zachary

6 A. Bright ${ }^{3}$, Russell A. Gould ${ }^{3}$, Olivia R. Ozguc ${ }^{2}$, Shawn T. O’Neil ${ }^{3}$, \& Molly Megraw ${ }^{1,3,4 *}$.

${ }^{1}$ Molecular and Cellular Biology Program, Oregon State University, 2701 SW Campus Way, Corvallis, OR 97331, USA.

$10{ }^{2}$ Biochemistry and Molecular Biology Program, Oregon State University, 2701 SW Campus

11 Way, Corvallis, OR 97331, USA.

$12{ }^{3}$ Department of Botany and Plant Pathology, Oregon State University, 2701 SW Campus Way, 13 Corvallis, OR 97331, USA.

$14{ }^{4}$ Center for Genome Research \& Biocomputing, Oregon State University, 2750 SW Campus

15 Way, Corvallis, OR 97331, USA.

Email: Molly Megraw* - megrawm@science.oregonstate.edu

${ }^{\dagger}$ Co-first authors

*Corresponding author

Running Title: Mining Tissue-Specific Gene Expression

Keywords: transcription factor / chromatin / gene regulation / Arabidopsis / machine learning

The author(s) responsible for distribution of materials integral to the findings presented in this

30 article in accordance with the policy described in the Instructions for Authors

31 (www.plantcell.org) is: Molly Megraw (megrawm@science.oregonstate.edu) 


\section{Abstract}

34 Across tissues, gene expression is regulated by a combination of determinants, including the

35 binding of transcription factors (TFs), along with other aspects of cellular state. Recent studies

36 emphasize the importance of both genetic and epigenetic states - TF binding sites and binding

37 site chromatin accessibility have emerged as potentially causal determinants of tissue specificity.

38 To investigate the relative contributions of these determinants, we constructed three genome-

39 scale datasets for both root and shoot tissues of the same Arabidopsis thaliana plants: TSS-seq

40 data to identify Transcription Start Sites, OC-seq data to identify regions of Open Chromatin,

41 and RNA-seq data to assess gene expression levels. For genes that are differentially expressed

42 between root and shoot, we constructed a machine learning model predicting tissue of expression

43 from chromatin accessibility and TF binding information upstream of TSS locations. The

44 resulting model was highly accurate (over $90 \%$ auROC and auPRC), and our analysis of model

45 contributions (feature weights) strongly suggests that patterns of TF binding sites within $\sim 500 \mathrm{nt}$

46 TSS-proximal regions are predominant explainers of tissue of expression in most cases. Thus, in

47 plants, cis-regulatory control of tissue-specific gene expression appears to be primarily

48 determined by TSS-proximal sequences, and rarely by distal enhancer-like accessible chromatin

49 regions. This study highlights the exciting future possibility of a native TF site-based design process for the tissue-specific targeting of plant gene promoters. 


\section{Introduction}

52 With the advent of genome-scale technologies, data has become increasingly available to address

53 the intriguing question of when and where a gene will express in multi-cellular organisms. Since

54 the entire genome of DNA sequence is identical in all of an organism's cells, what information

55 does RNA Polymerase II (pol-II) use to drive the transcription of many copies of a coding gene's

56 mRNA in one tissue or cell type, and very few copies in another? We know that "cause" is

57 ultimately connected to a complex series of interrelated events that determine cellular state at the

58 moments leading up to transcription initiation, including concentrations of various transcription

59 factors (TFs) and nucleosomes, DNA methylation states, and histone modification states.

60 Nonetheless, it is possible that DNA sequence alone contains all or most of the information

61 necessary to determine the tissues in which the gene will strongly express.

62 Previous studies have largely focused on chromatin state and available TF binding sites as

63 candidates for the primary determinants of tissue-specific gene expression, based on present

64 mechanistic understanding of pol-II transcription initiation. The depth of understanding of pol-II

65 promoter structure differs across multi-cellular eukaryotes (Smale and Kadonaga, 2003;

66 Kadonaga, 2004; Thomas and Chiang, 2006; Sandelin et al., 2007; Juven-Gershon and

67 Kadonaga, 2010; Kadonaga, 2012; Kumari and Ware, 2013) due to the timing of extensive

68 genome-scale data availability across species. Foundational studies in Drosophila have strongly

69 influenced concepts of 'core' promoter elements that reside at or immediately adjacent to the

70 transcription start site (TSS) and regulate basal transcription, 'proximal' regions that extend

71 beyond the core promoter but are also fundamentally important for transcription, and more distal

72 'enhancer' regions that are thought to regulate spatial and temporal control of transcription

73 (Kadonaga, 2004; Ong and Corces, 2011; Spitz and Furlong, 2012; Kumari and Ware, 2013).

74 While additional studies have broadened consideration of this paradigm over time particularly in

75 vertebrates (Andersson, 2015; Feuerborn and Cook, 2015; Kim and Shiekhattar, 2015) and plants

76 (Morton et al., 2014), still relatively little is known in many species about how 'core',

77 'proximal', and 'enhancer' regions are precisely defined genomically; the literature continues to

78 focus on TF binding sites in more TSS-distal enhancer-like regions as candidate master-

79 regulators of tissue specific gene expression (Ko et al., 2017).

80 The concept that 'accessible chromatin regions' or 'chromatin footprints' seem likely to pinpoint 
81 to specific regions of functionally bound TF sites, particularly in TSS-distal regions, has been

82 presented since the advent of genome-scale open chromatin studies (Heintzman et al., 2007; Xi

83 et al., 2007); this idea provides an attractive hypothesis that perhaps chromatin differences

84 between tissues or cell types 'modulate' the patterns of TF binding sites that are available,

85 thereby explaining gene expression differences between tissues. Many bioinformatic analyses

86 support various forms of correlation between patterns of open chromatin and gene expression in

87 a given tissue (Dong et al., 2012; Sheffield et al., 2013; Vera et al., 2014; Wilken et al., 2015;

88 Rodgers-Melnick et al., 2016; Snyder et al., 2016). A recent study in plants (Ricci et al., 2019)

89 specifically supports the idea that distal regions of open chromatin are statistically correlated

90 with tissue-specific gene expression, and that some of these regions are likely to be enriched for

91 relevant TF binding sites. Strikingly, however, there has been little evidence across the literature

92 that distal accessible chromatin regions are primary drivers of tissue-specific gene expression in

93 the case of most differentially expressed genes, or even that chromatin accessibility itself is

94 largely determining which TFs are able to bind and functionally interact with pol-II. In fact, a

95 recent study that includes mouse cells concludes in the title that "Accessibility of promoter DNA

96 is not the primary determinant of chromatin-mediated gene regulation" (Chereji et al., 2019). In

97 plants, studies comparing open chromatin landscapes across tissues and cell types (Zhang et al.,

98 2012b; Zhang et al., 2012a; Pajoro et al., 2014; Sullivan et al., 2014; Maher et al., 2018; Lu et

99 al., 2019) observe a surprising degree of qualitative similarity in general, including chromatin

100 patterns surrounding differentially expressed genes, and it is not clear whether more refined,

101 quantitative chromatin state differences may explain transcriptional program differences. The

102 primary determinants of gene expression in a given cell type or tissue thus remain a provocative

103 open question.

104 Machine learning models can potentially speak to this question by integrating genome-scale

105 datasets of different types- including both DNA sequence and chromatin state information- in

106 order to test whether enough information is present in these data types to predict tissue-of-

107 expression related outcomes. Several studies have specifically contributed to this line of inquiry

108 in the literature. An early study (Vandenbon and Nakai, 2010) used TF binding site information

109 to test whether DNA sequences alone could predict the cell or tissue type in which a gene would

110 be specifically expressed in 26 human and 34 mouse tissue and cell types. The study did this by 
111 training a series of models that would effectively predict whether a gene was more likely to

112 tissue-specifically express in one tissue vs another in the same species, with the highest inter-

113 tissue prediction success coming in at 73\% auROC for human Kidney vs Fetal Liver (auROC is a

114 performance measure of sensitivity and specificity, with a perfect model having auROC of $100 \%$

115 and a random classifier 50\%). This study did use TSS information to define promoter regions,

116 but high-precision genome scale TSS-sequencing data was not widely available at that time. A

117 study from several years later incorporated genome-scale OC data (Natarajan et al., 2012) to

118 examine 19 human cell lines, generating classifiers that predicted whether a gene would be

119 strongly upregulated in different cell types. Median performance was reported for a variety of

120 feature-generation techniques, the most successful technique achieved a median auROC of $73 \%$

121 by incorporating open chromatin information, with several of the top-performing models (out of

12219 models) achieving an auROC of nearly $90 \%$. Performance is not directly comparable with the

123 (Vandenbon and Nakai, 2010) study, because the goal was not to predict the tissue of expression

124 in a pair-wise setting but rather to distinguish genes that express very differently in a certain cell

125 type than in other cell types. Features were interpreted for well-performing models, and several

126 examples of tissue-specificity-associated TF binding locations within open chromatin were

127 observed to be important to the models in these cases. It was clear in this study that use of

128 chromatin information overall boosted performance, but it was very difficult to explain why just

129 a few models performed very well while others did poorly. This study used the single annotated

130 TSS location per gene, likely as genome-scale TSS-sequencing information was not available in

131 all cell types of interest at that time.

132 Recently, the study (Agarwal and Shendure, 2020) trained a deep-learning model with the

133 benefit of human ENCODE data that includes accurate transcription start site (TSS) locations in

134 each cell type, as well as mRNA stability data. This study focused primarily on modeling

135 transcript expression levels using TSS-sequencing data, but also trained a classifier to examine

136 whether the cell type could be correctly predicted for cell-type-specifically expressing genes in

137 human cell lines GM12878 and K562. The classifier achieved an auROC of 65\% in predicting

138 cell type from promoter sequence, but without use of TF binding site profiles of any kind. The

139 promising model performance success of all of these outcomes supports the idea that both TF

140 binding sites and chromatin accessibility carry considerable predictive power in classifying 
141 tissue of expression. Performance outcomes in the case of predicting tissue of expression in

142 inter-tissue or inter-cell-line comparisons remain relatively low compared to the high $80 \%$ 's

143 auROC that would be desirable for model interpretation. However, all of these past studies were

144 necessarily limited to available data sets that either did not have precise TSS and chromatin

145 information available in these same tissues, or - in cell line studies - cases where the material

146 under examination did not come from the same individuals and did not come from normally

147 functioning tissues. It was therefore not possible to inquire directly into the relative predictive

148 success contributions of primary sequence information and chromatin state with the benefit of

149 precise TSS locations in tissues or cells from the same healthy individuals.

150 This limitation may well have hindered predictive success in these past efforts, as precise TSS

151 locations are necessary to correctly define promoter sequences relative to the pol-II binding site.

152 TF binding sites are short (6-12nt) and sometimes degenerate sequences that appear throughout

153 the genome by statistical chance. Therefore, if one does not know the actual location of each

154 gene's highly expressing TSS(s) in a tissue, and instead 'guesstimates' for each gene with a

155 single annotated TSS (which is very likely to differ by at least 30-50 nt (nucleotides) from the

156 actual TSS by as much as $500 \mathrm{nt}$ in Arabidopsis (Morton et al., 2014)), then any 'promoter

157 sequence' under consideration may be shifted many binding sites away from the actual TSS-

158 proximal sequence. In this situation, not only is one unable to identify cases in which a different

159 promoter sequence is being used to transcribe a gene in a different tissue, but one is completely

160 unable to take advantage of accurate binding site patterns within each promoter-for example,

161 on a very simple level one cannot even know whether a TATA site seen $~ 25-35$ nt upstream of a

162 TSS is likely to be functional. Without the ability to approximate TSS location within a few

163 nucleotides, it simply isn't possible for a model to take advantage of precise patterns of

164 relationships surrounding these binding sites over thousands of TSSs expressed on the genome in

165 a given tissue sample. It will also be impossible to detect differences in these patterns for TSSs

166 expressed in a different tissue sample, omitting an important source of information, as pol-II can

167 utilize different transcription initiation locations for transcribing the same gene in different

168 organs (Forrest et al., 2014; Mejía-Guerra et al., 2015). In essence, because binding sites are

169 short and seen everywhere, important patterns in their relationships observed over thousands of

170 TSSs (in either the same or different tissues) can simply be 'washed away in the noise' if each of 
171 those TSSs is randomly shifted away from its actual location by many binding sites in genomic

172 distance.

173 In our study, we set out to construct a dataset that could help begin to quantitatively address the

174 informative components in pol-II gene promoters that explain tissue of expression. The large and

175 relatively complex yet well-studied genome of Arabidopsis, with many datasets from distinct

176 tissues/organs during plant development, presented an ideal organism in which to undertake this

177 task. We were able to construct a dataset from the same seedlings where each data component-

178 while using relatively new technologies at the time for Transcription Start Site Sequencing (TSS-

179 Seq) and Open Chromatin Sequencing (OC-Seq) — was able to be corroborated with other

180 published datasets derived from similar material, indicating some stability in these data with

181 regard to tissue/organ type, and allaying our concerns that our results might be particular to our

182 sample material or particular to the technology/protocol that we used to produce the different

183 dataset components. Our observations from machine learning model analysis of this dataset

184 challenged our previous assumptions that distal chromatin-accessible TF site locations play a

185 primary role in tissue of expression of most promoters, and suggest a possible paradigm shift in

186 the way we generally assume plant promoters to operate. Specifically, our findings suggest that

187 for the vast majority of differentially expressed genes in developing Arabidopsis organs, it is the

188 pattern of cis-regulatory sites in the TSS-proximal DNA of these regions, regardless of

189 chromatin state, that is most explanatory of the tissue of expression.

190 Results

191 TSS-Seq, RNA-Seq, and OC-Seq dataset in Arabidopsis roots and shoots captures 192 chromatin state together with promoter utilization in different plant organs

193 Our study uses "root" and "shoot" tissues harvested from 7-day old Arabidopsis thaliana

194 seedlings, dissected immediately below the hypocotyl (Figure 1). Each tissue batch was

195 separated into three portions, to which we applied Transcription Start Site Sequencing (TSS-

196 Seq), Open Chromatin Sequencing (OC-Seq), and RNA-Seq expression profiling protocols. We

197 used the nanoCAGE-XL protocol (Cumbie et al., 2015b) for performing TSS-Seq, the DNase-I-

198 SIM protocol (Filichkin and Megraw) for performing OC-Seq (Cumbie et al., 2015a), and

199 applied a standard RNA-Seq protocol (see "Dataset Generation" section in Methods). The

200 nanoCAGE-XL and DNase-I-SIM protocols were developed by the lab to work efficiently with 

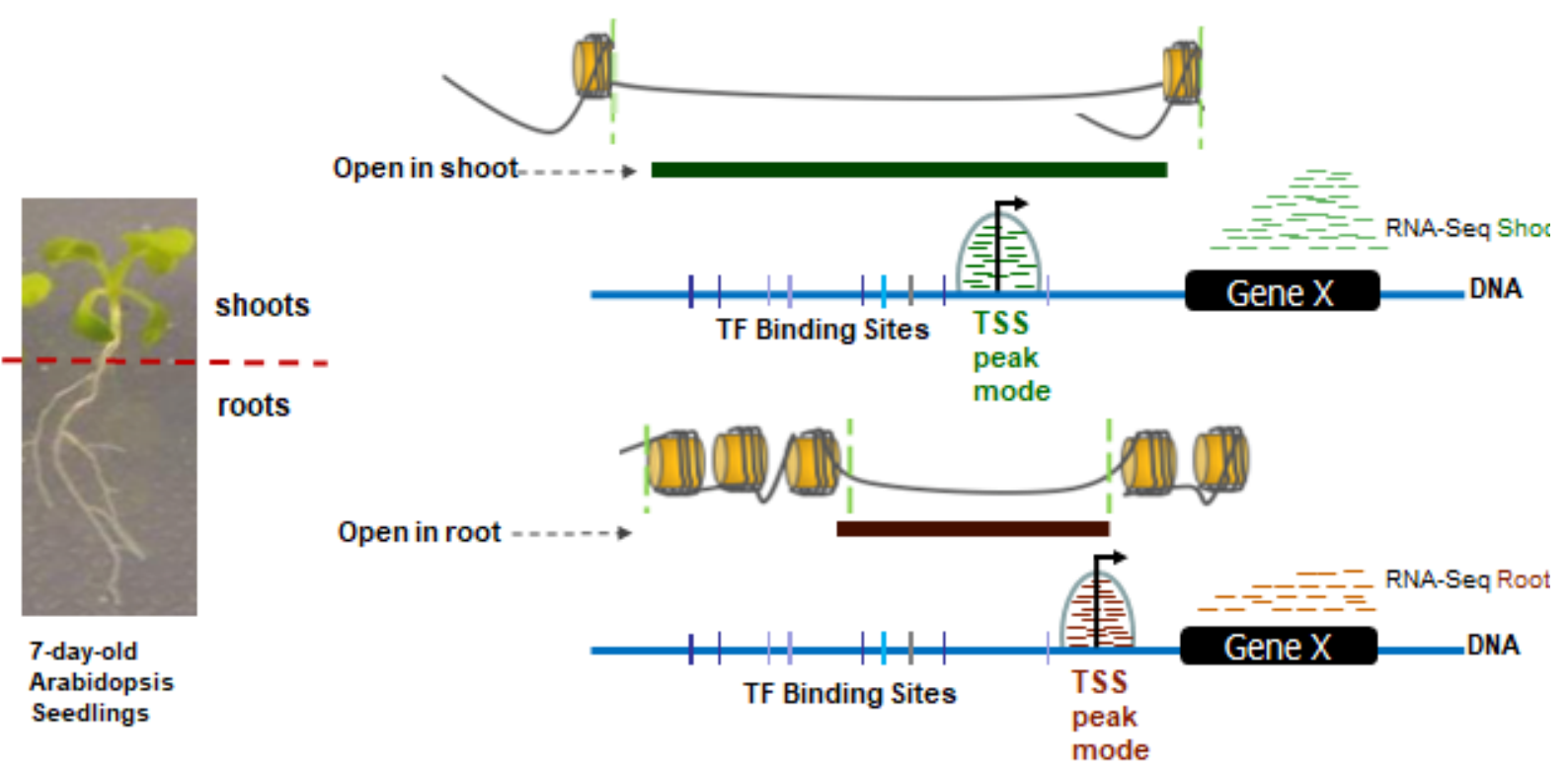

Figure 1. Datasets generated from 7-day-old wildtype Arabidopsis thaliana Columbia 0 roots and shoots. RNA-Seq reads align to annotated gene bodies to demonstrate gene expression, while OC-Seq reads highlight DNase-I Hypersensitive Sites. TSS-Seq reads align in peaks around the TSS; the mode of the peak is designated the location of the TSS.

202 relatively low-volume plant tissues such as Arabidopsis seedling roots and shoots; these were

203 vetted in publication using datasets that were generated by applying other current protocols to

204 comparable tissue samples sequenced on the Hi-Seq 2000, which sequenced to sufficient depth

205 and coverage to support our study (see Supplementary Figure 1). We also noted that outcomes

206 for both nanoCAGE-XL and DNase-I-SIM were remarkably consistent with other TSS-Seq

207 datasets (Morton et al., 2014) and DNase-I-Seq datasets (Zhang et al., 2012a) generated in

208 similarly prepared Arabidopsis seedling tissue samples.

209 Figure 1 illustrates the data collection goal of our study. As described in the Introduction, precise

210 TSSs are critical to any predictive modeling effort that seeks to relate TF binding sites to gene

211 expression outcomes, particularly those in different tissues, organs, or cell types. Clearly a

212 reasonable estimate of highly accessible or "open" chromatin locations is also critical to our

213 query, as it is plausible that TF binding sites within open chromatin regions are playing a large

214 role in tissue-specific gene expression. Finally, we gathered RNA-Seq data for our samples as

215 this form of expression profiling provides the most well-studied statistically robust estimate for

216 gene expression levels, despite its implicit 3' locational bias (Ross et al., 2013). We used these 
A

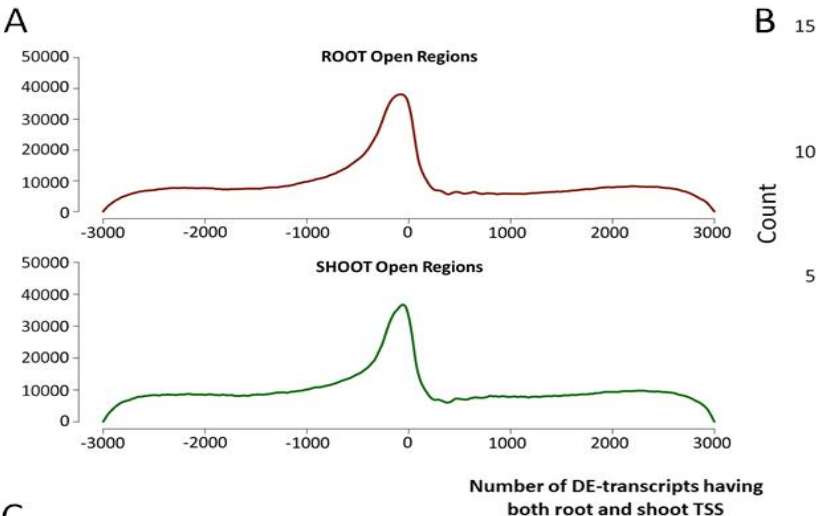

C

$\mathrm{D}$

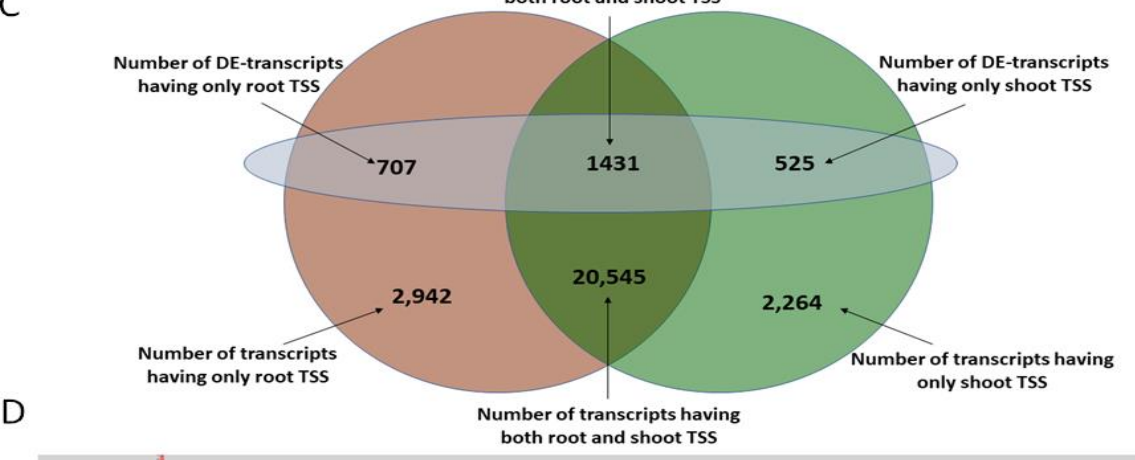

Figure 2. Summary of data outcomes. A) Charts comparing the general accessibility in roots (top) and shoots (bottom). B) C) Bar chart showing the difference in location between transcriptassociated TSS modes in root and shoot. The majority of TSSs have similar locations in the two tissues, but $\sim 16 \%$ have very different locations (>100 nt). C) Numbers in the top row of the Venn diagram represent differentially expressed (DE) transcripts, separated by tissue in which the transcripts have associated TSS peaks. The numbers at the bottom represent all transcripts associated with TSS peaks, separated by tissue in which they have TSS peaks. Total number of DE transcripts $=2,663$; total number of transcripts $=24,928$. D) A sample gene displayed in 
218 three data types to take an initial survey of apparent differences in gene expression program in

219 our root and shoot samples (Supplementary Tables $1-4)$. Although we observed that TSS

220 location and promoter accessibility are quite similar across tissues for many genes (Figure 2A),

2212663 transcripts show strong expression in only one of the two tissue types (Figure 2C).

222 Additionally, 525 differentially expressed transcripts are associated with a TSS peak in shoot

223 only, while 707 transcripts have a TSS peak in root only. The 1431 differentially expressed

224 transcripts with TSS peaks in both tissues results in 1632 peak pairs. Of these pairs, $222(\sim 14 \%)$

225 have very different TSS mode locations (TSS-mode-distance > $100 \mathrm{bp}$ ), and 471 cases have

226 mode locations that differ by 10 to $100 \mathrm{nt}$. Out of the 939 cases which have a very similar TSS

227 mode location (TSS-mode-distance < 10), we looked for differences in patterns of open

228 chromatin (\% nucleotides disagreeing in chromatin state, Supplementary Figure 2). In the vast

229 majority of cases, we were intrigued to see very little obvious difference in chromatin state-that

230 is, we saw a large overlap in the percent of nucleotides agreeing in accessibility state. We

231 observed that while major differences in TSS location or chromatin state might simply explain

232 differential expression between the two tissues in perhaps $20 \%$ of the cases, in most cases the

233 reason for differential expression could not be attributed to any obvious difference either in TF

234 binding site usage or chromatin accessibility. We concluded that a quantitative modeling effort

235 was necessary for further investigation.

236 Highly expressed TSSs can be accurately modeled in each tissue type using only DNA 237 sequence

238 Past machine learning studies have shown that strongly expressing TSS locations in a tissue 239 sample can be precisely predicted from DNA sequence alone, using surrounding TF binding sites 240 as 'features' - that is, numerical descriptors of the genomic location that one is inquiring about 241 with the question "Is this location a highly expressing TSS or not" (Megraw et al., 2009; Morton 242 et al., 2014). We hypothesized that if one could accurately model highly expressing TSS

243 locations in the root sample and in the shoot sample individually, using TF binding site (TFBS)

244 information as features, one could then inquire into model differences in binding site patterns 245 that are "important to root expression" vs those that are "important to shoot expression". For this 246 task, we selected the 3PEAT model (Morton et al., 2014), as it remains the only high-

247 performance plant TSS peak finder to date with features that can explicitly be interpreted as 
representing TF:promoter binding site interactions. Additionally, the 3PEAT model had previously been applied to an Arabidopsis root sample grown under nearly identical conditions to those in our current study, but where the sample was generated using a different TSS-Seq protocol known as "Paired-End Analysis of Transcription start sites" or "PEAT" (Ni et al.,

252 2010); this enabled us to understand whether our nanoCAGE-XL TSS-Seq datasets would

253 support a similarly successful model to the PEAT root sample, which achieved an auROC in the 254 high 90\%'s. We applied the 3PEAT model to both root and shoot nanoCAGE-XL TSS-Seq 255 samples from our study and found that we could predict strongly expressing root and shoot TSS 256 locations in independent single-tissue-type models each with an auROC of $98 \%$.

257 We then examined the TFs associated with the top-weighted features (TF binding locations most 258 important to model success) of the trained root and shoot 3PEAT models (Supplementary Table

2595 , Supplemental Data Set 1), to determine whether there were any obvious root-specific or shoot260 specific differences. We observed that the two models shared the majority of their top-50 most 261 important TFs, though a few differences in the top-10 indicated the possibility of a more 262 important role for root-development-related TFs in the root model and shoot-development263 related TFs in the shoot model (Supplementary Figure 3). We then looked for quantitative 264 evidence of root-specific vs shoot-specific TF binding site pattern usage in the two models by 265 applying the root-trained model to the shoot model's test set (i.e. locations that are either highly 266 expressed TSSs or not highly expressed in shoot), and the shoot-trained model to the root 267 model's test set (i.e. locations that are either highly expressed TSSs or not highly expressed in 268 root) (see Methods for details). Surprisingly, both models performed essentially identically on 269 test sets of TSSs in the 'other' tissue as on test sets of TSSs from the tissue in which the model 270 was trained (Supplementary Table 6) - with the same 98\% auROC and only a negligible drop 271 below 80\% in auPRC (area under the Precision Recall Curve, a complementary performance 272 measure). However, we found a tendency for the models to produce approximately $20 \%$ more 273 false positives (classifying non-TSS sites as TSSs) when applied to the test set derived from the 274 'other' tissue compared to the model that was trained on that tissue. This was compensated by an $2758 \%$ decrease in false negatives by the shoot-trained model on the root test set compared to the 276 root-trained model and a 15\% decrease in false negatives by the root-trained model on the shoot 277 test set compared to the shoot-trained model (Supplementary Figure 4). Additionally, genes with 
TSSs that were misclassified by the shoot-trained model were statistically enriched for several root development GO-terms as compared to the full set of peaks that were tested (see Methods, Supplementary Table 7). Several additional GO-term enrichment and depletion observations, taken together with model feature-weight observations, strongly supported the idea that patterns of TF binding sites were likely to be well-explaining TSS expression in both tissues; yet the core of both models almost certainly described sequence information indicative of general transcription, as opposed to tissue-specific expression. We concluded that the 3PEAT TSS prediction concept provided an appropriate feature set that would potentially allow us to model the differences in tissue of expression based on TF binding site information, but would need to be incorporated into a model that focused on differentially expressing genes.

\section{TSSs enable meaningful TF binding-site-based feature set construction}

289 The original 3PEAT model investigation (Morton et al., 2014) demonstrated that precise TSS

290 locations were key to training a highly accurate TSS prediction model, with a substantial $\sim 10 \%$

291 auROC performance drop if only annotated start sites were used. We wanted to investigate

292 whether the 3PEAT model's TF binding site-based feature set construction was not only the key

293 to predictive success in explaining strong TSS expression, but also carried plausible support for

294 explaining pol-II transcription in reality. Specifically, we wanted to test whether the putative

295 binding sites modeled as important to a gene's correct TSS location prediction were also likely to

296 be TF-bound in the sample, therefore potentially functional. We also wanted to gain a basic

297 indication of whether 'predictive sites' extracted from model features - that is, important TF

298 binding site-enriched regions known in the PEAT model as "Regions of Enrichment" or ROEs

299 (Figure 4B) - were likely to be highly sensitive to the specific dataset in terms of sample

300 collection, TSS-Seq protocol, or informatic processing details such as selection of peak caller

301 parameters. We selected the root sample for testing, as it was then possible to compare 3PEAT

302 TSS models built from two different datasets using very similar tissue samples, (i) the PEAT

303 dataset (Morton et al., 2014) and (ii) the nanoCAGE-XL dataset generated for the present study.

304 We began by selecting putative cis-regulatory sites from the original 3PEAT model application

305 to the PEAT dataset for in vitro TF protein:DNA binding interaction testing using the following

306 procedure (see "Functional Binding Site Selection" in Methods for details). Cis-regulatory

307 elements considered by the model included TF binding sites as well as core promoter elements 
such as TATA-box which facilitate direct interactions with the pol-II complex. First, likelihood scores for individual putative binding sites that contributed to each TSS prediction (i.e. each

310 transcript detected in the Arabidopsis root) were calculated using their corresponding Positional

311 Weight Matrix (PWM) binding domain representation and their position relative to the ROE for

312 each element. The output of this pipeline was a genome-wide "master-list" of potentially

313 functional cis-regulatory sites. These candidates were filtered by considering only strongly

314 expressing "narrow-peak" TSSs, which have an enriched association with developmental genes

315 responsible for tissue-specific patterning (Morton et al., 2014), and other restrictions to generate

316 a stringent short-list of 500 sites (i.e. sites associated with the top $20 \%$ by importance-rank

317 according to their 3PEAT model weight, then sites with highest likelihood scores located near

318 the center of their ROE and within 120bp of their corresponding TSS). Finally, we selected five

319 "high-scoring” candidate sites (INI-B, TATA box, Y-Patch, PIF3-binding element, and

320 SQUAMOSA Promoter Binding element SQUA1) for evaluation using the Electrophoretic

321 Mobility Shift Assay (EMSA or "gel shift" assay) and nuclear extracts prepared from

322 Arabidopsis roots. We included several sites from the HSP90.2 promoter, one of the few genes

A

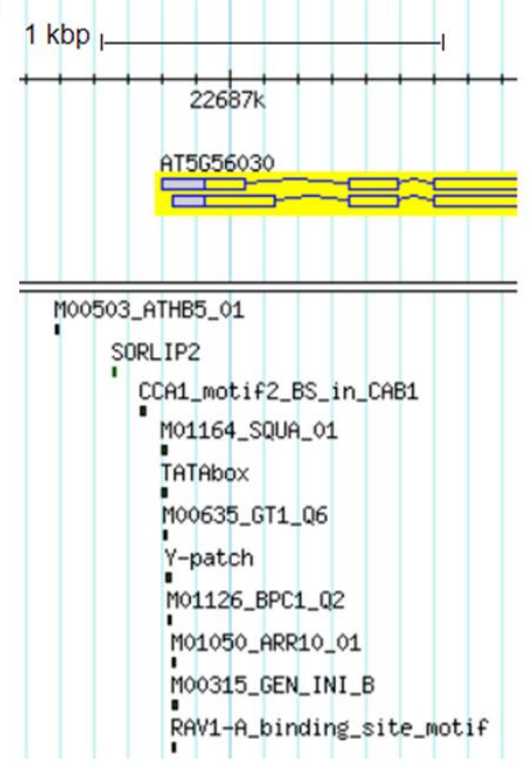

B

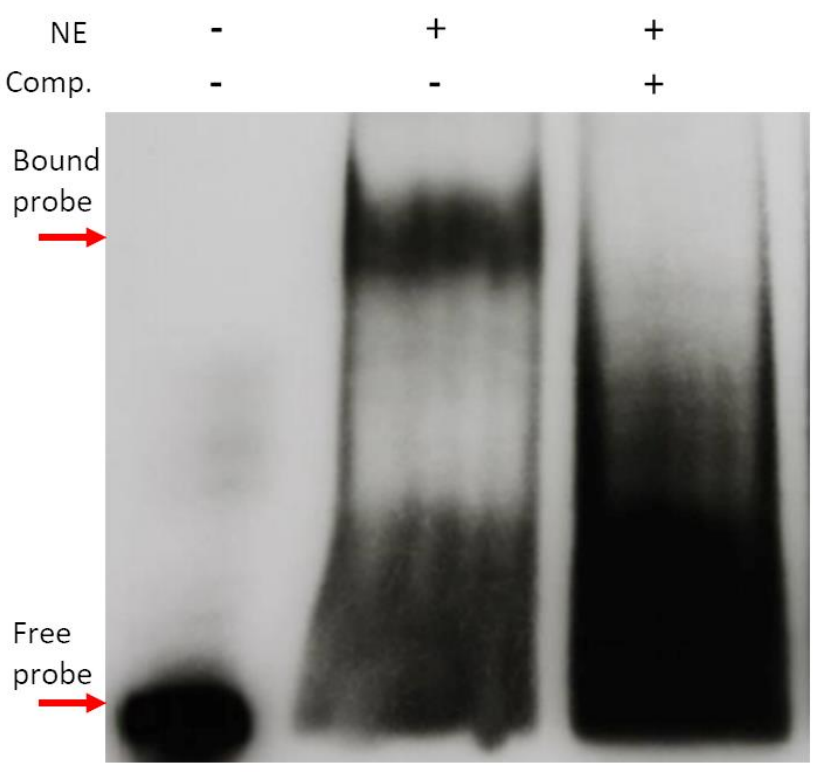

Figure 3. Selection and testing of putative functional binding sites. A) The HSP90.2 (AT5G56030) promoter region in GBrowse. Locations of the tested transcription factor binding sites in this promoter are displayed below the gene model. B) Y-patch electrophoretic mobility shift assay. The presence of a shifted band of probe indicates higher molecular weight than the free probe due to TF binding. The right-most lane contains $>200 \mathrm{X}$ cold competitor to show that the shift is not an artifact. 
324 with top-ranked sites in our list that had a known function, as well as one site each from the

325 promoters of ornithine carbamoyltransferase (OTC) and diacylglycerol kinase 2 (DGK2); in this

326 first selection, we focused on sites that were located in regions of open chromatin in root, but did

327 not account for expression level of the TF(s) corresponding to the PWM binding domain profile

328 predicted to target the candidate sites. We observed gel-shifts for four out of the five candidate

329 sites.

330 In troubleshooting the case that did not shift, we observed that PIF3, a circadian-controlled TF,

331 had a very low level of expression as measured by the RNA-Seq outcome in the root sample of

332 our current study. We also observed a general qualitative correlation between the intensity of the

333 shifted band and RNA-Seq expression level with the other candidates, suggesting that TF

334 binding would be undetectable below a certain level in nuclear extracts. We then selected 6

335 additional top-scoring sites in the HSP90.2 promoter (Figure 3) but filtered out any sites

336 associated with very lowly expressed TFs as measured by our RNA-Seq sample. Of these sites,

337 all 6 resulted in a shifted band, indicating binding. We then considered whether applying the

338 same process to the 3PEAT model, when trained using more recent peak-calling on the

339 nanoCAGE-XL root sample, would include these same sites as relatively important to the

340 expression of the target gene. We repeated the process ("3PEAT-style Model Construction" in

341 Methods) using the 3PEAT model re-trained on the nanoCAGE-XL root sample that achieved an

342 auROC of $98 \%$ described in the section above. We observed that all of our selected sites in the

343 HSP90.2 promoter were included in the new top sites list, although some had a lower score; the

344 tested site in the DGK2 promoter does not appear on our new list, though this gene had a very

345 weak TSS peak in the sample, consistent with involvement in circadian function including lack

346 of upregulation by PIF3. OTC had a moderate TSS peak in the sample, and its site was included

347 (Supplementary Figure 5). In considering whether additional information could be obtained by

348 performing traditional gel-shifts for these sites using purified TF protein, we concluded that if

349 successful this would only demonstrate the ability to bind an oligo at unrealistically high

350 concentrations of each TF, essentially confirming the TF's PWM binding domain description as

351 provided by a database. In total, the gel-shift of 10/11 predicted binding sites using nuclear

352 extracts, taken together with qualitative correspondence between RNA-Seq level of the candidate

353 TF and darkness of the shifted band, as well as the relatively stable predictive importance of 
354 these sites across TSS-Seq datasets, provided plausible support for binding of these sites at in

355 vivo TF concentrations. We concluded that 3PEAT model's ROE-based TF binding site features

356 represent sites that are at least potentially functionally bound in a way that promotes their target

357 gene's transcription by pol-II.

358 TFBS locations and their chromatin state accurately predict tissue of expression for 359 differentially expressed genes

360 Building on the successful TF Regions of Enrichment feature concept of the 3PEAT model for

361 predicting TSS location, we constructed an analogous model that we called the Tissue of

362 Expression Prediction ROE model or TEP-ROE model. We reasoned that if patterns of TF

363 binding site enrichments can predict the locations of strongly expressing TSSs on the genome,

364 and high-affinity binding sites within important enrichment regions are plausibly functionally

365 contributing to pol-II's frequent transcription initiation at these locations, then perhaps it is

366 patterns of TF binding sites within these regions that can help well-distinguish a tissue in which a

367 gene will express strongly from a tissue in which it will express to a much lesser extent. But it

368 also seemed that the general accessibility of sites in these regions could prove important, as

369 could general sequence enrichments such as AT-content and overall degree of openness in the

370 vicinity of the TSS. Figure 4A shows the concept of the TEP-ROE model, with details provided

371 in Methods. Like 3PEAT, TEP-ROE is an L1-regularized logistic regression classifier that takes

372 as input (i) the DNA sequence surrounding a TSS (TSS - $1 \mathrm{~kb}$, TSS + $500 \mathrm{nt}$ ) and (ii) chromatin

373 accessibility state for both tissues in this region around the TSS, and returns the predicted tissue

374 (root or shoot) in which that TSS will express most strongly. The most important concepts for

375 understanding and interpreting the model (Figure 4A) are that (1) each TFBS feature represents a

376 specific genomic region in relationship to a TSS where a particular TF binding domain has a

377 high density of high affinity binding sites, (2) each OC feature represents the "openness" (degree

378 of accessibility) of a corresponding TFBS feature region, as a percent of accessible nucleotides

379 in this region, (3) the two OC_overall features OC_overall_root and OC_overall_shoot represent

380 the percent openness of the 'proximal' region [TSS - $500 \mathrm{nt}$, TSS + $100 \mathrm{nt}$ ] around a TSS in root

381 and in shoot, (4) sequence enrichment features (e.g. GC Content) represent the percent of certain

382 nucleotides (e.g. G and C) in a $100 \mathrm{nt}$ window around the TSS, and (5) the weight that a

383 successfully trained model gives to each of these features represents an 'importance value' - a 
A

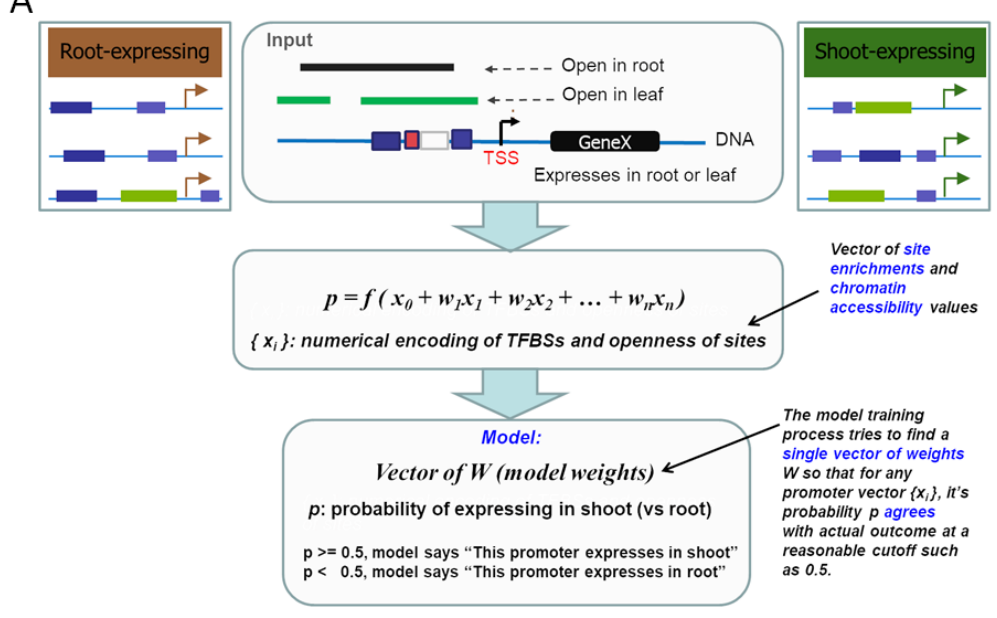

B

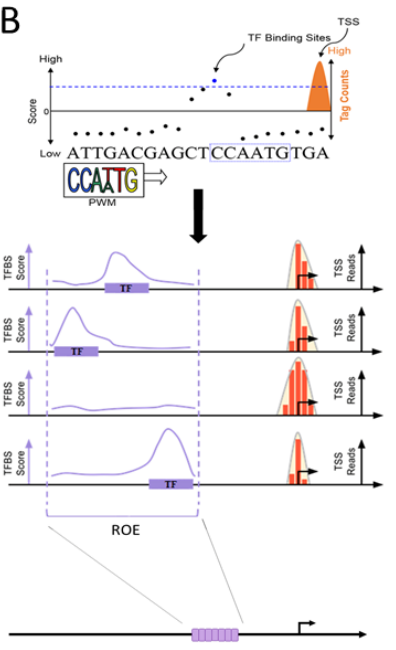

C

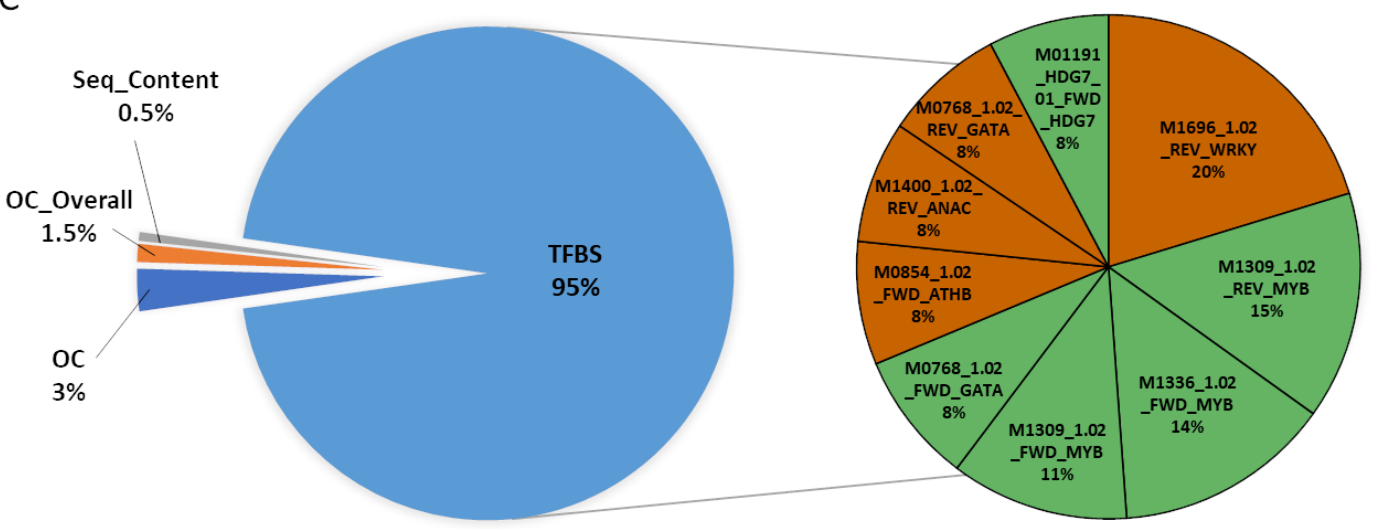

Figure 4. A) TEP model concept: Three data types (TSS-Seq, OC-Seq, RNA-Seq) are generated from roots and shoots; this allows us to numerically encode TFBS presence and chromatin accessibility. The numerical encoding is then used to train and test a machine learning model that outputs the probability of a transcript's expression in root or in shoot. B) TEP-ROE feature generation: Regions of enrichment (ROEs) are detected by scanning a PWM (TF binding profile) over each promoter region and calculating where the TFBS loglikelihood scores are significantly higher than background levels. These regions are then further divided into windows for feature scoring. C) In the chart to the left, TFBS features make up $95 \%$ of the total generated. The chart on the right highlights the features that the TEP-ROE model weighted most heavily. The names of these features contain quite a bit of relevant information (e.g. M1969_1.02_REV_WRKY: M1969_1.02 is the PWM designation from the database; REV indicates that the feature is located on the opposite strand from the gene; and WRKY is the associated TF family). Green pie wedges indicate that the model deemed this feature important for expression in shoots, while orange wedges indicate importance for expression in roots.

385 large weight magnitude or "top-ranked feature" indicates a feature whose value contributes heavily toward the decision about whether a TSS is predicted to express strongly in root or 
strongly in shoot.

388 We trained the TEP-ROE model on TSS locations associated with differentially expressed genes, 389 using cross-validation for parameter selection and an independent held-out test set for reporting 390 test performance (see "Model Training and Testing on nanoCAGE-XL TSSs" section in

391 Methods). The model achieved an auROC of $92 \%$ and an auPRC (area under the Precision

392 Recall Curve, an important co-indicator of performance) of 94\% (Supplementary Figure 6). In

393 order to evaluate performance stability over a wide variety of dataset divisions (training vs

394 testing) and algorithm seedings (different initial value settings of the optimization algorithm), we

395 re-trained the model 30 times with different 'seeds' (Supplementary Figure 9). We observed that

396 auROC and auPRC model performance outcomes were tightly distributed around means which

397 were close to our TEP-ROE model's performance values and concluded that our TEP-ROE

398 model's strong performance was representative. We then examined feature stability by looking at

399 the feature weight ranking distribution for each of the 50 top-ranked features in the model, over

400 the 30 models used in performance stability testing (Supplementary Figure 10); we found feature

401 rankings to be acceptably stable in the sense that each of the 50 most important features stayed

402 within the top-ranked 50 for all other test models, and most features' rank remained within 5-10

403 ranking slots of the mean in the vast majority of the test models.

404 Performance and stability indicated that it was meaningful to interpret the TEP-ROE model, as

405 top-weighted features were likely to be important contributors to successful tissue prediction.

406 The two OC_overall features were high-ranked contributors (Figure 4C, Supplemental Data Set

407 2), with a few general sequence content features falling into the top 300. The most striking aspect

408 of the model outcome is that aside from the OC_overall features for root and shoot, and a small

409 number of sequence content features, TFBS features comprised all of the top 350 features, with

410 the first OC feature appearing at rank 352. We performed a literature search on the top 100 TFBS

411 feature binding domains, and found that of the 20 which had functional annotation, four had

412 literature support for activity in the same tissue whose weight sign (positive or negative)

413 indicated that presence of this TFBS site density made expression in this tissue more likely. The

414 locations of these important regions fell within $500 \mathrm{nt}$ of the TSS, indicating the strong predictive

415 role of TF site densities in this proximal region. Finally, when we re-trained a version of the

416 TEP-ROE model using only the TAIR10 annotated start site for each differentially expressing 
417 gene rather than TSS-Seq peak locations, auROC dropped to 76\%. This substantial $\sim 15 \%$

418 auROC performance drop supports an important role for precise TSS locations in successful

419 tissue of expression prediction model training.

420 Promoter 'tiling' model offers complementary view of important feature locations

421 The TEP-ROE model was constructed around the concept of "Regions of Enrichment", which

422 are special regions that one can think of as containing "high TF binding site densities" for a

423 particular TF with respect to all TSSs in a sample type. Since this model style focuses only on a

424 single Region of Enrichment for each TF, and not all TFs have these high binding site densities

425 in our root and shoot samples, some TFs and their binding sites are omitted from consideration in

426 the TEP-ROE model. We also wondered if the TEP-ROE concept was unnecessarily "confining"

427 important TF binding patterns that are considered by this model to locations very near to the

428 TSS, just because this is where the highest binding site densities occur for most TFs. This lead us

429 to ask whether a model that simply "tiled" the same region surrounding the TSS with "tile

430 regions' (Figure 5A) would achieve similar or even greater performance - and if it did, would

431 such a model select similar TF binding site density regions as important features. We constructed

432 the TEP-Tiled model by following an identical procedure to the TEP-ROE model, except that

433 ROEs were replaced by a series of non-overlapping $100 \mathrm{nt}$ windows tiling the entire [TSS - $1 \mathrm{~kb}$,

434 TSS + $500 \mathrm{nt}$ ] region under consideration (see "Promoter Tiling" section in Methods).

435 The TEP-Tiled model achieved nearly identical performance results (Supplementary Figure 7) to

436 the TEP-ROE model, and its performance over a large number of seeded trials was similarly

437 stable (Supplementary Figure 9). Building the model using only annotated TSSs from TAIR10

438 caused a similar $\sim 15 \%$ auROC performance drop to that of the ROE model. The stability of top-

439 weighted features decreased as compared to the TEP-ROE model (Supplementary Figure 11),

440 but this is largely to be expected because the TEP-Tiled model has many thousands of additional

441 features (many more tiles than ROE regions) and is therefore a very highly under-constrained

442 model; that is, there are so many more features whose importance the model must consider than

443 there are TSSs in the root and shoot classes that (1) there are many feature combinations that can

444 potentially help the model to perform well, and (2) the model operates at the limit of the

445 regularization process' ability to identify meaningful feature combinations. It is this second issue

446 that lead to declining performance when we examined models using tiles smaller than $100 \mathrm{nt}$ 

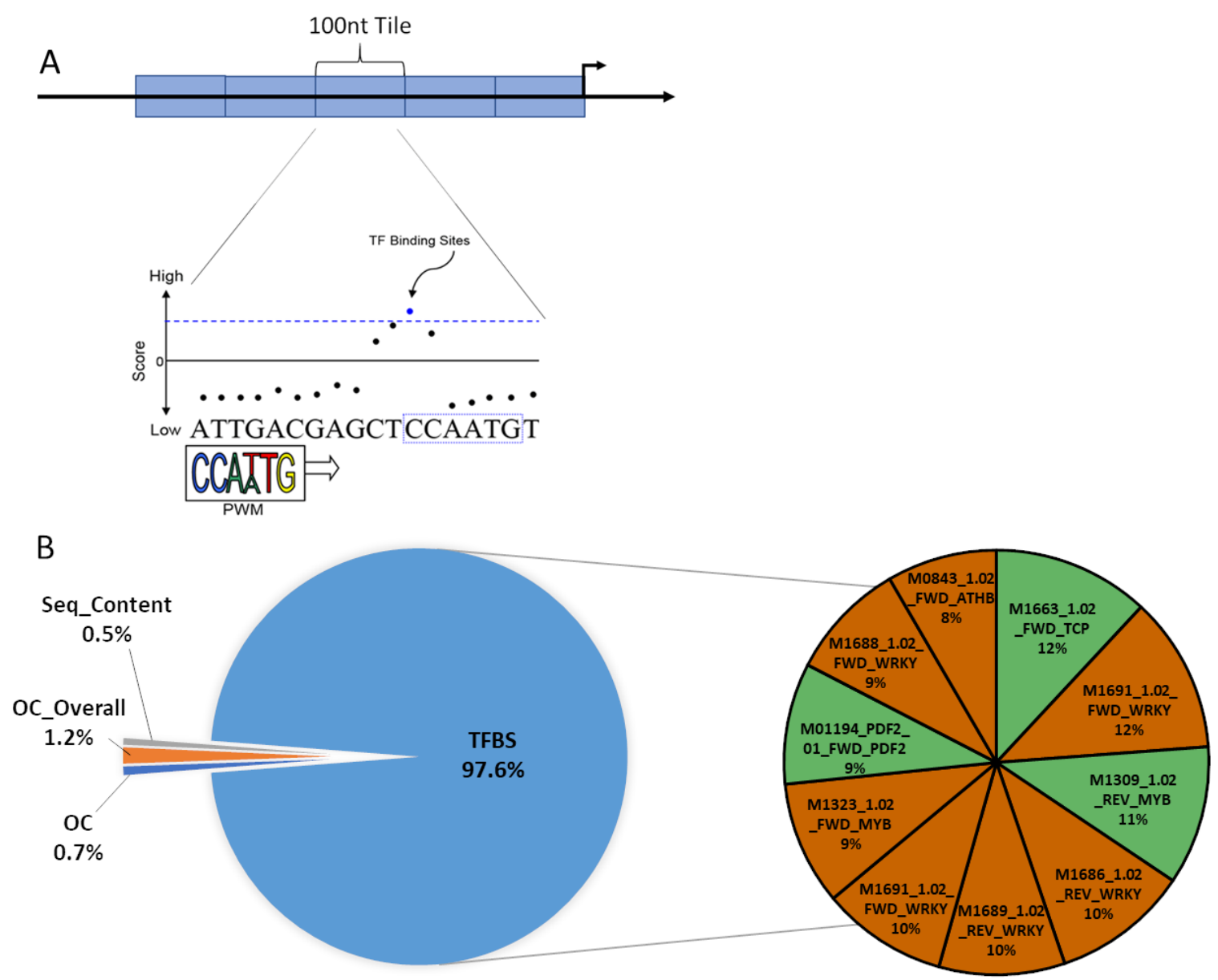

Figure 5. TEP-Tiled model. A) Cartoon schematic of TEP-Tiled model's feature generation. Instead of identifying ROEs and creating features within these smaller regions, this model generates $100 \mathrm{nt}$ wide tiles over the entire promoter. B) As with the TEP-ROE model, TFBS features comprise the majority of the features generated by the TEP-Tiled model (97.5\%). The pie chart to the right contains the top 10 most heavily weighted features. As with the TEP-ROE model, feature names have three parts (e.g. M1691_1.02_FWD_WRKY: M1961_1.02 is the database's identifier for the PWM; FWD means the feature is on the same strand as the gene; and WRKY is the associated TF family). Green pie wedges are features that the model deemed important for expression in shoots and the orange pie wedges are features the model deemed important for expression in roots.

449 meaningfully examine the type and location of its most important features (Figure 5B). The

450 OC_overall features play a similarly important role, and sequence content features have similar

451 rankings in general. The first TFBS-associated OC region appears at a lower importance rank

452 ( 740) than in the TEP-ROE model ( 350), as TFBS features even more heavily dominated the 453 top importance weight rankings. 
454 To visually examine the relationships between top-ranked TFBS feature locations in the TEP-

455 Tiled and TEP-ROE models, Figure 6 shows a heatmap overlay of the 100 top-weighted features

456 in each model, displayed according to location with respect to the TSS. In general, for most of

457 the TFBS features that the two models agree are in the top 100 (those rows that contain both red

458 and blue hues), there is some form of 'telescoping effect' or overlap in the regions that both

459 models consider highly important. In these cases, typically the Tiled model agrees with at least

460 one of the locations that the ROE model considers most important for a TF binding domain type,

461 but also gives some lesser weight to at least one additional location. This seems to suggest that

462 much of the time, when the models agree on an important TF binding domain, there is a

463 tendency to agree on its most important location. But, clearly, there are many 'only red' or 'only

464 blue' rows indicating that there is agreement on inclusion of a TF binding domain feature only

465 about one third of the time. Supplementary Table 8 provides a quantitative look at whether the

466 two models agree on what the most important TFBS or OC features are, location aside. In

467 considering the top 10-weighted features in each model, about 30\% are shared. However, all

468 disagreements in this case appear to result from selection of different members of the same TF

469 family by each model (among TF-associated features), as M1691_1.02_TFBS,

470 M1686_1.02_TFBS, M1696_1.02_TFBS are all WRKY family transcription factor binding

471 domains. In general, 20-30\% features are identical between the two models in considering up to

472200 top-weighted features, and dissimilarities appear to be due at least in part to the different

473 models' inclusion of non-identical but relatively numerically similar binding domain profiles

474 among TF families.

475 Finally, while Figure 6 shows that the most important locations in both models tend to fall within

$476500 \mathrm{nt}$ of the TSS, the TEP-Tiled model indicates that occasionally an important TF binding

477 domain location could be located nearly $1 \mathrm{~kb}$ upstream of the TSS. The most important ROEs

478 from the TEP-ROE model all lie within $500 \mathrm{nt}$ of the TSS, but we wondered if the TEP-Tiled

479 model would select important tiles more than $1 \mathrm{~kb}$ upstream if given the opportunity. We re-

480 trained the TEP-tiled model using [TSS - $2 \mathrm{~kb}$, TSS + $500 \mathrm{nt}$ ] and observed a slight performance

481 drop, with no top-10-ranked tiles in importance falling upstream of $1 \mathrm{~kb}$ (Supplementary Figure

482 14, Supplementary Table 9). Overall, comparisons between models support the idea that the TF

483 binding locations which contribute most to model performance - that is, to the model's ability to 
correctly predict the tissue of expression-lie within about $500 \mathrm{nt}$ of the TSS.

TEP models suggest some promoters may express almost solely based on patterns of functionally bound sites

487 In examining the target genes of TFs that were identified by a model as very important to differential expression, we observed that the promoters of some TSSs seemed to contain highaffinity binding site densities in important regions for several different TFs. We were interested to investigate whether some promoters seemed to be "hard-coded", in the sense that the promoter's associated tissue of expression appeared to be entirely dictated by TF binding site patterns, with no influence from chromatin state according to a successfully trained Tissue of

493 Expression Prediction (TEP) model. We ran an analysis to identify promoters where tissue of expression prediction was successful and nearly all of the promoter's 'important feature products'-meaning high-affinity TF binding site densities (TFBS features) or large chromatin accessibility values (OC features) that were associated with high model weights (feature importance values) — derived almost entirely from the presence of high-affinity TF binding site densities. We identified promoters for 18 genes that fell above the $95^{\text {th }}$ percentile for 'hardcodedness' (see Methods) using the TEP-ROE model, and 43 genes using the TEP-Tiled model

500 (Supplementary Tables 10 and 11). Both gene sets were enriched for GO terms associated with metabolite biosynthesis and transport (Supplementary Table 12), while the TEP-Tiled model 'hard-coded' genes were additionally associated with development.

The models suggest that genes whose promoters are 'hard-coded' by TF binding site content to express differentially in roots vs shoots (or vice versa) could be preferentially involved in very basic processes that need to be performed differently in one tissue vs another during development, based on TF presence alone. This implies that chromatin state in these cases is perhaps directly modulated by one or more of the TFs involved in the important binding site patterns; we did observe that in the case of each model, at least one 'top TF binding domain' associated with the most important feature products was known to be involved in chromatin

510 remodeling (Supplementary Table 12).

511 As a result of these inquiries, we wondered whether there existed cases of promoters such that

512 'zeroing out' a single TF binding site density would be predicted to 'flip' the tissue in which a

513 gene was most highly expressed. The physical analog of this experiment would be a form of 'in21 
514 silico knockout', where high-affinity binding sites within an important region for a TF's

515 influence on tissue of expression are removed or occluded, so that this TF can no longer bind in

516 this region with respect to the current TSS. We observed that the TEP-ROE model points to 8

517 genes whose promoters contain 'knockout regions' that would cause a 50\% probability shift 

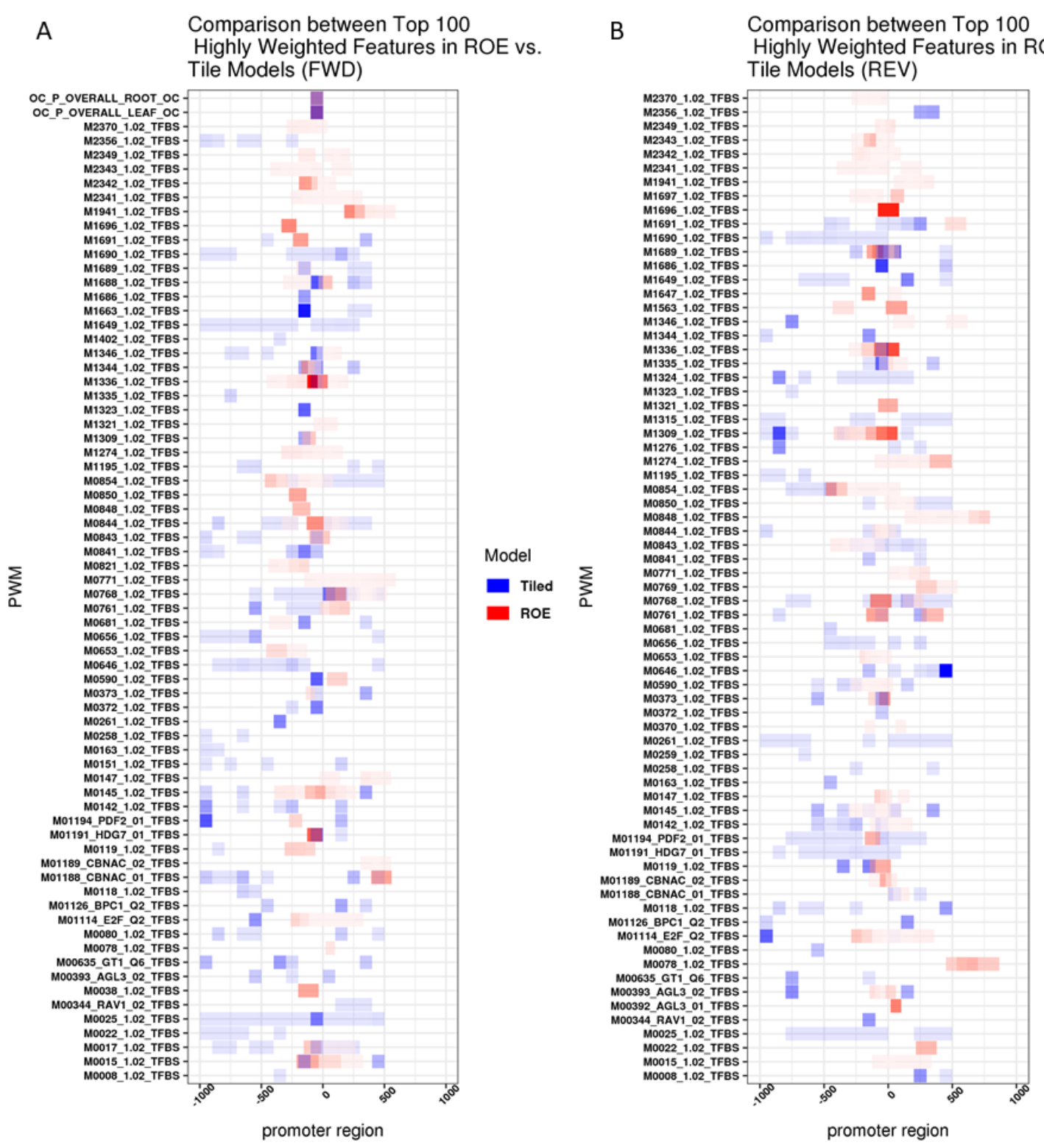

Figure 6. Comparisons of top PWMs between TEP models. A) Heatmap showing the differences between and shared PWMs on the same-as-gene (FWD) strand that are weighted highly by TEP-ROE (red) and TEP-Tiled (blue). B) Heatmap showing the differences between and shared PWMs on the opposite-from-gene (REV) strand that are weighted highly by TEP-ROE (red) and TEP-Tiled (blue).abase's identifier for the PWM; FWD means the feature is on the same strand as the gene; and WRKY is the associated TF family). Green pie wedges are features that the model deemed important for expression in shoots and the orange pie wedges are features the model deemed important for expression in roots.

519 'across the decision boundary' to change the predicted tissue of strongest expression

520 (Supplementary Table 13). The TEP-Tiled model, which has many more regions than the TEP-

521 ROE model, points to 28 such TF-tile 'knockout' locations that cause at least $50 \%$ probability 
522 shifts or greater to 'flip' predicted tissue of strongest expression (Supplementary Table 14). For

523 the TEP models, several of these tissue-flip-causing TF binding site density 'knockout' regions

524 were also among the top TF binding domains important to 'hard-coded' promoters. This outcome

525 appears to corroborate the presence of highly influential binding region locations for specific TFs

526 that may be serving in an important 'master regulatory' role for the tissue of expression of some

527 promoters. Collectively, modeling experiments suggest that it is typically pairs or larger groups

528 of these important TF binding density regions located in spatial patterns that most heavily

529 influence tissue of expression within the $\sim 500 \mathrm{nt}$ upstream proximal promoter region of a

530 transcript.

\section{TF site presence and location are predominant explainers of tissue of expression}

532 Although TFBS features were highly dominant in both the TEP-ROE and TEP-Tiled models, we

533 unexpectedly observed that the chromatin regions surrounding the important TFBS feature

534 binding site density locations were not considered important at all by the model. In fact, TFBS

535 feature weight values assigned by each TEP model had virtually no correlation with model

536 weight (importance) of the corresponding region of chromatin (Supplementary Figures 15 and

537 16). Additionally, we observed no correlation between the importance of regions containing

538 large TFBS densities within individual TSS promoters and chromatin openness in these regions

539 (Supplementary Figures 17 and 18). This was perplexing; because model structure means that

540 important TFBS features represent locations where large TFBS densities contribute strongly to

541 expression in one tissue vs another, we had anticipated that the state of chromatin accessibility of

542 these regions at least in some cases would be correspondingly important. We noted that the

543 OC_overall features, representing openness of the general proximal [TSS $-500 \mathrm{nt}$, TSS + 100

$544 \mathrm{nt}$ ] region, received high weight in both tissues for both models. Both models also agreed on

545 TFBS features as overwhelmingly more important than OC features as a collection. It seemed

546 possible then that a higher degree of general openness of this TSS-proximal region was

547 contributing the vast majority of chromatin state information in the models.

548 We decided to examine whether removal of OC features entirely would seriously hurt model

549 performance. We therefore re-trained both the TEP-ROE and TEP-Tiled models on feature sets

550 that were identical to the original models with the exclusion of any OC feature. We observed a

$551 \sim 5 \%$ drop in auROC in both cases (Figure 7), with the resulting "TFBS-Only" models 
552 performing surprisingly strongly at $87 \%$ auROC, with $88 \%$ and $91 \%$ auPRC respectively. We

553 then wondered if a TFBS-Only model with root and shoot OC_overall features included, but no

554 other OC features, would perform as well as the original model. We tested this idea, and both

555 model types achieved essentially the same performance as the original TEP-ROE and TEP-Tiled

556 models achieved with all feature types present (Figure 7). Thus, by returning only a single

557 general measure of openness of the [TSS - $500 \mathrm{nt}$, TSS + $100 \mathrm{nt}$ ] proximal promoter region in

558 each tissue to a DNA-sequence-based-features-only version of

\begin{tabular}{|c|c|c|c|}
\hline Moc & & auROC & auPRC \\
\hline TFBSs + Chromatin state $-\mathrm{ROE}$ & $\overline{7}$ Gene- & $92 \%$ & $94 \%$ \\
\hline TFBSs only - ROE & 내낸 Gene- & $87 \%$ & $88 \%$ \\
\hline Chromatin state only - ROE & Gene- & $84 \%$ & $86 \%$ \\
\hline TFBSs + OC General - ROE & 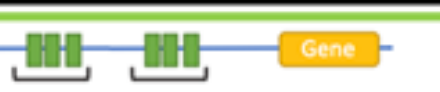 & $91 \%$ & $92 \%$ \\
\hline TFBSs + Chromatin state - Tiled & 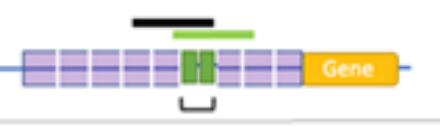 & $93 \%$ & $94 \%$ \\
\hline TFBSs only - Tiled & 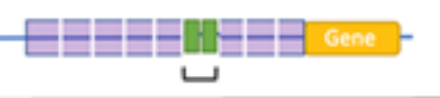 & $87 \%$ & $91 \%$ \\
\hline Chromatin state only -Tiled & 프프프픜ㅋ & $85 \%$ & $88 \%$ \\
\hline TFBSs + OC General - Tiled & 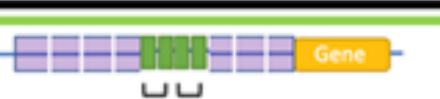 & $91 \%$ & $93 \%$ \\
\hline
\end{tabular}

Figure 7. Performance summary. The removal of chromatin information from our feature set results in a performance decrease of 5-6\% in both of our TEP model types. Removal of sequence-based information decreases performance by $8-9 \%$ in the TEP models. Addition of a general openness feature for the promoter region to the TFBS only TEP models (blue-highlighted lines) restores performance essentially to the same level as the original models containing all chromatin features.

560 each model, performance was restored to essentially the same level as when all OC features were 561 included. 
562 Finally, we expected that the 'inverse' experiment-- removal of all TFBS features-- would not

563 seriously hurt model performance, because there is a strong tendency of expressing promoters to

564 be more open (Figure 2A) within $\sim 1 \mathrm{~kb}$ upstream or so of the TSS; thus, open chromatin in

565 general should be a strong predictor of differential gene expression. We went forward with this

566 experiment and observed that both models performed with an auROC of $\sim 85 \%$ (Figure 7), a 7\%

567 drop in auROC from the original TEP-ROE and TEP-Tiled models. This result was surprising

568 only in that it suggests that chromatin-state patterns, at least on a regional scale, can predict

569 tissue of expression in strongly differentially expressing cases only about as well as TFBS and

570 other DNA sequence content information alone. By far, the highest weighted coefficients in this

571 model were the OC_overall root and shoot coefficients, corroborating the predictive power of

572 general chromatin openness within the [TSS - $500 \mathrm{nt}, \mathrm{TSS}+100 \mathrm{nt}$ ] region.

573 While it is possible that examination of OC patterns on a binding-site-scale might contribute

574 some additional information if it were to become technically feasible to train such a model, all

575 outcomes in our set of experiments clearly suggest that TF binding site information within

576 promoter DNA is the predominant explainer of the tissue of expression. In particular, outcomes

577 support the concept that patterns of TFBS densities within the 'more-open' 500 nt proximal

578 promoter region provide the largest influence on the tissue(s) in which a gene will preferentially

579 express. Outcomes support a secondarily influential role for the degree to which a promoter is

580 generally open in this region within a tissue, although of course the modeling experiments cannot

581 inform whether this higher degree of proximal-promoter openness results from a chromatin

582 remodeling process that is TF-dependent. Surprisingly, modeling outcomes did not support any

583 direct association between important TF site density locations and the importance of chromatin

584 state in these locations.

\section{Discussion}

586 Model success in predicting tissue of expression fundamentally derives from precise TSS

587 information

588 The Tissue-of-Expression-Prediction or "TEP" models constructed in our study are conceptually 589 straightforward classical machine learning models - they use L1-regularized logistic regression

590 to find specific high-affinity TF binding site regions positioned in relationship to a TSS, along

591 with chromatin accessibility values in these regions, that collectively identify the correct tissue of 26 
592

593

594

595

596

597

598

599

600

601

602

603

604

605

606

607

608

609

610

611

612

613

614

615

616

617

618

619

620

621

greater expression for the vast majority of differentially expressed genes in our sample set (auROC $\sim 90 \%$ ). Previous attempts at this specific task in similarly genomically complex organisms have made good progress but achieved middling results at best (auROC 75\%). Why then did the TEP models perform so well as compared to past models, including a recent deep learning model in cell lines (Agarwal and Shendure, 2020) that did in fact have precise genomewide TSS-Seq information available? The construction of a first-of-its-kind dataset with generation of both Transcription Start Site sequencing data and Open Chromatin sequencing data in two different tissues of the same healthy individuals likely contributed to success. However, all of our experiments clearly indicate that the most important contributor to predictive success was modeling with accurate TSSs in each plant organ. Our outcome is consistent with similar classical machine learning studies including (Vandenbon and Nakai, 2010) in demonstrating that TF binding site information alone is capable of achieving relatively strong predictive performance, and (Natarajan et al., 2012) in confirming that chromatin accessibility information boosts inference of genes that are expressing differently in different tissues/cell-types. In relationship to the (Vandenbon and Nakai, 2010) study, which performed an identical task with auROC of $75 \%$, the TEP models' $\sim 15 \%$ auROC performance drop when only annotated start sites were used is consistent with the idea that accurate TSS information within each tissue is largely responsible for dramatic performance boost.

However, precise TSS information alone is unlikely to be solely responsible for high sensitivity and specificity given that a sophisticated DNA-sequenced-based deep learning model had the benefit of TSS-seq data but achieved only $\sim 65 \%$ auROC on this task; (Agarwal and Shendure, 2020) concludes in fact that the model's performance is not boosted by the use of TSS-seq data instead of annotated start sites. Given the observations in our study, it is very likely that explicit use of important biological information such as TF binding profiles in feature set construction confers a large benefit in predicting tissue of expression. TEP model feature sets are carefully constructed to use high-affinity TF binding site densities as opposed to thresholding, and to encompass positional relationships of these densities to the TSS. Additionally, despite its relative simplicity as a classical machine learning model, regularized logistic regression is a time-tested method that still routinely outperforms deep-learning approaches in genomic classification of phenotype from transcriptomics data (Smith et al., 2020). While deep learning models hold 
622 exciting promise, it seems likely that current architectures are as-yet unable to learn TF binding

623 site features with enough precision to take advantage of patterns in their positional relationships

624 to each other and to the TSS.

625 We would hypothesize in this context that much of the "missing mass" in performance to bring 626 auROC up near 100\% with a TEP-style model is contained in the incomplete TF binding site 627 domain profile collection presently available even in a well-characterized model species such as

628 Arabidopsis. It is certainly possible that an unconsidered influence such as DNA methylation

629 status plays a role, although this seems largely correlative with chromatin status and not

630 necessarily definitively causal. Finally, it is possible that important micro-scale chromatin

631 accessibility patterns are not able to be well-captured at present by our model, given that the

632 potentially relevant set of binding site locations and their associated chromatin state is enormous

633 as compared to the set of highly expressed TSS locations in the genome; regularization

634 algorithms do have limits on their ability to select the most predictive features from a vast sea

635 uninformative values using a relatively small number of examples. Yet we see little indication of 636 this 'micro-scale chromatin accessibility pattern' transcriptional control concept within the fairly

637 broad regions of accessible chromatin in the Arabidopsis proximal promoter.

638 Accurate TSSs implicate proximal cis-regulatory regions as primary determinants of 639 tissue-specific gene expression

640 Our modeling outcomes strongly suggest that DNA sequence, within about 500 nt directly

641 upstream of the TSS, is by far the most influential feature in successfully predicting tissue

642 expression level differences, as opposed to distal chromatin status. Specifically, our study

643 suggests a paradigm shift in the way we generally assume plant promoters to operate: for the vast

644 majority of differentially expressed genes in developing Arabidopsis organs, it is the pattern of

645 cis-regulatory sites in the TSS-proximal DNA of these regions, regardless of chromatin state,

646 that is most explanatory of the tissue of expression. The presence of TF binding site Regions of

647 Enrichment, and the ability to predict both TSS location and tissue of expression primarily from

648 binding sites within these regions, underscore the important tissue specificity role of TF binding

649 site patterns within the [TSS - $500 \mathrm{nt}$, TSS + $100 \mathrm{nt}$ ] 'proximal' promoter region in developing

650 Arabidopsis seedlings.

651 It is surprising that TF binding site patterns in relatively accessible proximal promoter regions 28 
652 could largely dictate a gene's tissue of expression, though there is a growing body of genome-

653 scale evidence that this may well be the case in higher eukaryotes (Vandenbon and Nakai, 2010;

654 Huminiecki and Horbańczuk, 2017; Chereji et al., 2019). Studies such as (Maher et al., 2018)

655 emphasize specific groups of TFs that appear to act as 'control modules' within different plant

656 tissues and cell types; it may be the case that a relatively small and distinct group of TF master

657 regulators tends to work in-concert within each tissue to help orchestrate chromatin remodeling,

658 ensuring that promoter regions are largely accessible in the important proximal locations.

659 Additionally, several studies suggest the intriguing possibility that distal enhancer regions may in

660 fact be playing a significant role in tissue specific gene expression, but that Pol-II interactions

661 with these enhancers are dictated to a high degree by proximal promoter sequences. The (Taher

662 et al., 2013) study entitled "Sequence signatures extracted from proximal promoters can be used

663 to predict distal enhancers" provides substantive computational evidence for this concept. (Ong

664 and Corces, 2011) provides a literature synthesis of studies on enhancer function in tissue

665 specific gene regulation, noting cumulative evidence that chromatin looping between enhancer

666 and promoter regions is likely to be dictated at least in part by specific groups of TFs. Our study

667 is consistent with the possibility that distal enhancers are indeed playing a substantial role, but

668 are interacting with specific patterns of TFs which bind the proximal promoter to mediate

669 chromatin looping.

\section{Implications for synthetic biology: systematic design of tissue-specific promoters}

671 A recent study (Cai et al., 2020) strongly supports the concept that the specific locational

672 arrangements of endogenous binding sites within a plant promoter can have a dramatic effect on

673 overall expression level. The construction and outcomes from our Tissue-of-Expression-

674 Prediction from Regions of Enrichment or "TEP-ROE” model carry two practical implications

675 along these lines for additionally directing strong expression in one tissue vs another. Firstly, the

676 TEP-ROE model identifies specific TSS-proximal TF binding site regions as important to

677 differential gene expression in each tissue sample - in our study, developing Arabidopsis roots

678 and shoots. Our gel-shift analysis provides plausible support for the idea that when these model-

679 identified patterns of high-affinity TF sites are located upstream of a specific promoter, then

680 these sites may be bound and functional, serving in the context of surrounding sequence to

681 preferentially upregulate gene expression in a particular tissue. Secondly, when certain TF 
682 binding densities are given a zero-coefficient or 'removed' from a promoter, this can produce a

683 large shift across the decision boundary, indicating a model prediction that removal of high-

684 affinity sites in this region would change the tissue in which a gene expresses most strongly.

685 In other words, in-silico "knockouts" identify TF binding regions that alter the predicted tissue in 686 which the gene is differentially expressed. There are hundreds of cases in which a single TF687 region 'knockout' is predicted to cause such a shift, and thousands of cases in which a 'double-

688 knockout' is predicted to cause such a shift. Taken together, these results suggest strong potential 689 for tissue-specific promoter design. For this application, rather than focusing on differentially 690 expressed genes, one would re-train a TEP-ROE model to classify the tissue of expression for 691 genes that expressed very highly in one tissue and very little in the other, in terms of absolute 692 transcript counts. This would allow identification of specific high-affinity TF binding sites that, 693 when removed from the context of a certain promoter, change the tissue of expression for a gene 694 entirely. In summary, our model presents the exciting possibility that tissue-specific synthetic 695 promoters can be systematically constructed using endogenous cis-regulatory sites whose 696 presence/absence in specific locations leads to a predicted shift in tissue of expression.

\section{Methods}

\section{Plant materials and sample preparation}

699 Arabidopsis thaliana ecotype Columbia 0 seeds were sterilized in a solution of 50\% (v/v) bleach 700 solution with $0.1 \%$ Tween 20 for 10 minutes, then rinsed extensively with sterile deionized

701 water. Sterilized 100 micron nylon mesh was placed on top of solidified medium (30 mM

702 sucrose, 4.2 g Murashige and Skoog medium (PhytoTech Labs), and $0.8 \%$ Phytagar, pH

703 adjusted to 5.8 with $\mathrm{KOH}$ ) in large petri plates (Genesee Scientific). Following a 4 day

704 vernalization period in water, sterilized seeds were suspended in a $0.75 \%$ agar solution and were 705 transferred to each plate in two dense rows ( $\sim 500$ seeds per row) in a laminar flow hood under

706 sterile conditions. Seedlings were grown vertically in a Conviron PGR15 growth chamber at

$70721^{\circ} \mathrm{C}$ under a 12:12 hour light:dark cycle (50\% humidity, and $250 \mathrm{~mol} / \mathrm{m} 2 / \mathrm{s} \mathrm{light} \mathrm{intensity).} \mathrm{At}$

708 seven days, the seedlings were harvested and divided into three batches. For each batch,

709 seedlings were dissected using a surgical blade and the root tissue was separated from the shoot 710 tissue. For our purposes here, shoot tissues include the hypocotyl, cotelydons, and any stems and 
711 true leaves that had developed by the time of collection. Each batch of seedlings was handled

712 identically, and harvested tissues were flash-frozen in liquid nitrogen, then stored at ${ }^{-} 80^{\circ} \mathrm{C}$ until

713 needed for the following protocols.

\section{Dataset generation}

\section{Sequencing}

716 TSS-Seq was performed for both root and shoot samples as described in (Cumbie et al., 2015b)

717 using the nanoCAGE-XL protocol in conjunction with the HiSeq-2000 sequencing platform. For

718 DNase-seq, chromatin from isolated nuclei was digested with DNase I and libraries were

719 prepared for both root and shoot samples according to the DNase-I-SIM protocol (Filichkin and

720 Megraw) as published in (Cumbie et al., 2015a). RNA was isolated from both root and shoot

721 samples for RNA-Seq using the RNeasy kit (Qiagen). Samples were analyzed for quality on the

722 Bioanalyzer 2100 (Agilent) and only RNA with a RIN > 9.0 was used. Single-end

723 libraries were sequenced on the Illumina HiSeq-2000 sequencing platform in triplicate.

\section{Read preprocessing and alignment}

725 CapFilter software (Cumbie et al., 2015b) was used to pre-process all nanoCAGE-XL TSS

726 sequence files prior to alignment, removing library artifacts such as extra guanines at the

727 beginning of the reads. For all three sequencing experiments, single-end reads were aligned to

728 the TAIR10 reference genome (Lamesch et al., 2012), using Bowtie version 2.0 (Langmead and

729 Salzberg, 2012) with the parameter settings '-v 0 -m 1 -a best strata' (uniquely mapped reads

730 with only one mismatch allowed).

731 nanoCAGE-XL TSS-Seq data processing

732 After cap-filtering and aligning the TSS-Seq reads for the root and shoot samples, the JAMM

733 peak finder (Ibrahim et al., 2015) was used to identify TSS read clusters. For TSS datasets in our

734 study, the fragment size and bin size were both set to 10. The output of JAMM is a list of peaks

735 along with their genomic coordinates. The coverage subcommand from the bedtools software

736 suite (Quinlan and Hall, 2010) was used with the parameter settings -s (requiring same-

737 strandedness) and - $d$ (for reporting depth at each position) to retrieve the number of aligned reads

738 in each peak region for peak annotation. An R script was developed to process the aligned reads

739 within peak regions and generate peak information, such as the number of aligned reads in peak, 
740 TSS peak mode location, and mode read count. Each TSS peak was assigned to the closest

741 TAIR10 annotated transcript, and peaks which fell within 250 bps upstream of the annotated

742 translation start site and contained more than 50 read counts were selected for use.

\section{DNase I SIM data processing}

744 After alignment to the genome, the F-Seq peak-calling software (Boyle et al., 2008) was used to 745 identify DNase-I hypersensitive sites (DHSs), as in (Cumbie et al., 2015a). We chose this OC-

746 Seq peak caller because it provides compatible output with the original DNASE-I ENCODE 747 data, and is therefore comparable with DNase-I peak usage by the machine learning modeling 748 studies discussed in the Introduction and Discussion sections. Subsequent peak callers have more

749 parameters that can be tuned, but all peak callers are smoothing algorithms that have limitations 750 and tradeoffs in signal processing parameter selection. F-Seq was run with a specified feature 751 length of 300 and a minimum DHS length of $50 \mathrm{nt}$.

\section{RNA-Seq data processing and Differential Expression Analysis}

753 Individual transcript abundance was determined using the RSEM software package (Li and 754 Dewey, 2011) for each root and shoot RNA-seq sample. RSEM enables accurate transcript 755 quantification using its built-in bowtie 2 alignment by building the reference sequence from a 756 user-provided genome annotation and calculating expression for each isoform. We used rsem757 prepare-reference and rsem-calculate-expression for preparing reference sequence and 758 computing transcript abundances, respectively. We then used EBseq (Leng et al., 2013), which is

759 included in the RSEM package and is robust to outliers, in order to detect differentially

760 expressed transcripts (rsem-run-ebseq).

\section{$761 \quad$ TSS-Seq data quality analysis}

762 Sequence depth analysis

763 To determine whether our sampling depth was sufficient to accurately represent gene expression

764 in root and shoot samples, we performed saturation analysis on our nanoCAGE-XL data. This 765 analysis was performed as described in (Morton et al., 2014). Supplementary Figure 1 contains 766 the results of this analysis. 


\section{PEAT-style Models}

768 Model construction

769 Using our nanoCAGE-XL peak data, we constructed models to predict whether a given genomic

770 location is a TSS for each tissue type. Starting with annotated peaks, we removed those with less

771 than 100 reads per peak or less than 30 reads at the peakmode, and kept only those peaks labeled

772 as being a TSS, within the 5'UTR region, or within 500 nucleotides of the TSS. We then

773 generated the model features from TAIR10 sequences surrounding these peak regions using the

774 TFBScanner (Morton and Megraw, 2014). The filtered peaks were then used to train and test

775 3PEAT models exactly as described in (Morton et al., 2014).

\section{Functional Binding Site Selection}

777 The TSBS sequence features, their model-assigned weights, and the TSS probabilities generated

778 from the root-trained model were then used to construct a table of putative functional binding

779 sites and their associated metrics. This table was then filtered to retain only Narrow Peak

780 promoters. Next, the data was sorted by model output probability, descending total feature score,

781 descending model weight, descending false negative rate, ascending false positive rate, peak

782 count read, and the absolute value of relative location. TFBS sites were selected from the top 500

783 rows of this sorted table for wet-lab validation of functional binding.

784 EMSA

785 Nuclear extracts were purified from Arabisodpsis thaliana Columbia 0 roots following a slightly

786 modified protocol from (Staiger et al., 1991). We spun our cellular lysates at $2200 \times \mathrm{g}$ for 1

787 minute at $4{ }^{\circ} \mathrm{C}$ before passing the supernatant through a series of progressively finer meshes (100

788 micron, 60 micron, 30 micron). Nuclei were washed and pelleted at $2200 \mathrm{x} g$ for 10 minutes at

$7894^{\circ} \mathrm{C}$. Halt Protease Inhibitor Cocktail (Thermo Fisher Scientific) was used in place of the $\mathrm{KCl}$ in

790 Buffer B. After dialysis, samples were prepared with the Qubit Protein Assay Kit (Invitrogen)

791 per manufacturer instructions and total protein concentration was measured on a Qubit

792 Fluorometer (Invitrogen). DNA probes were designed using selected PWMs and the flanking

793 sequences from the associated TSS. Both the template strand and its reverse complement were

794 labeled using the Pierce Biotin 3' End Labeling Kit (Thermo Fisher Scientific) and the

795 complementary oligonucleotides were annealed. EMSAs were performed using the LightShift

796 Chemiluminescent EMSA kit (Thermo Fisher Scientific). Briefly, $15 \mu \mathrm{L}$ of nuclear extract 
797 (containing $4 \mu \mathrm{g}$ total protein) was incubated together at room temperature for 30 min with $79880 \mathrm{fmol}$ of biotin-labeled probes in $25 \mu \mathrm{L}$ reaction mixtures containing $1 \mathrm{X}$ Binding Buffer, 10 $799 \mathrm{mM}$ DTT, $40 \mathrm{ng} / \mu \mathrm{L}$ poly $(\mathrm{dI}-\mathrm{dC})$, and $2 \%(\mathrm{v} / \mathrm{v})$ glycerol and then separated on $6 \%$ native

800 polyacrylamide gels in Tris-borate-EDTA buffer containing $45 \mathrm{mM}$ Tris, $45 \mathrm{mM}$ boric acid, and $8011 \mathrm{mM}$ EDTA, pH 8.3. Unlabeled probe was used as cold competitor in 300X excess. Labeled 802 probes were detected using the Pierce Chemiluminescence Detection Kit (Thermo Fisher

803 Scientific) according to manufacturer instructions and visualized on an Azure c600 imager 804 (Azure Biosystems).

805 Evaluation of Peak Classification in Same-Tissue and Other-Tissue Datasets

806 Two 3PEAT style models were constructed - one trained on $80 \%$ of the nanoCAGE-XL peaks

807 from root and the other on $80 \%$ of the peaks from shoot. Models were tested on the other $20 \%$ of

808 the peaks from both the same tissue and the opposite tissue. Then, we determined which peaks

809 were misclassified by the models. For each test set, we divided the peaks which were classified

810 incorrectly into four groups: "root-misclassified" (misclassified only by the root-trained model),

811 "shoot-misclassified" (misclassified only by the shoot-trained model), "both-misclassified"

812 (misclassified by both models), and "any-misclassified" (includes all peaks misclassified by at

813 least one of the models). For each test-set-model pair, we compiled a list of genes that had at

814 least one TSS peak which was misclassified by only that model. We observed that these lists

815 greatly overlapped with the other list of their respective tissue-type, therefore we chose to

816 exclude genes that were common to both lists. For each list of genes described above, we

817 performed a GO enrichment analysis using GOATOOLS (Klopfenstein et al., 2018), a Python-

818 based automated gene ontology enrichment analyzer. We used the genes represented in the full

819 test set pertaining to the list as a population for comparison, limiting the scope of the analysis to

820 "Biological Process" ontology terms, and limiting results to those with $\mathrm{p}<0.05$. Additionally, a

821 simple Plant-Ontology enrichment analysis (Jaiswal et al., 2005) was performed by creating a list

822 of terms subordinate to each of the terms of interest, "root system" (PO:0025025), and "shoot

823 system" (PO: 0009006). Genes with peaks misclassified exclusively by one of the models, which

824 were annotated with terms subordinate to one of the terms of interest were considered to be

825 matches to those terms. A Fisher's exact test was performed comparing the proportion of

826 matches in genes misclassified by one model exclusively vs. genes misclassified by either model, 
827 with a cutoff of $\mathrm{p}<0.05$ for significant enrichment or depletion.

828 Tissue of Expression Prediction Modelling

829 Construction of TEP models

830 Feature Generation

831 Each TFBS feature represents an approximation of cumulative binding affinity that a particular

832 TF has for a specific genomic region. Each open chromatin feature represents a percentage of

833 nucleotides within the associated region that are open. In the model versions that used TFBS

834 features, each ROE region as determined in previous section is divided into five overlapping sub-

835 widows and two flanking windows as described in (Morton et al., 2014). The TFBS features are

836 cumulative log-likelihood scores for each ROE sub-window on the same and opposite strands (as

837 the gene in consideration). The chromatin features are computed as the percentage overlap

838 between the open regions and each ROE sub-window. Only log-likelihood scores greater than

839 zero are considered as potential binding sites and contribute to the sum, therefore the minimum

840 value for a TFBS feature is 0 (this is a case where none of the nucleotides in the region represent

841 a potential binding site with a greater-than-zero log-likelihood score). In the Tiled model, the

842 entire region from $1 \mathrm{~kb}$ upstream to 500bp downstream of the TSS mode is divided into non-

843 overlapping windows of $100 \mathrm{bp}$ in width. The TFBS features are computed as cumulative log-

844 likelihood scores within each tile for both strands. The open chromatin features for Tiled model

845 are computed as a percentage overlap between the open regions and each tile. In addition to

846 TFBS and chromatin features, we added sequence content features such as GCcontent (CG\%

847 within 100 bp upstream of TSS mode), CAcontent (CA\% within 100 bp upstream of TSS mode),

848 GAcontent (GA\% within 100 bp upstream of TSS mode), ATcontent, and general promoter

849 openness. ATcontent features were computed for each $20 \mathrm{bp}$ tiles within -200 to +40 bps from the

850 TSS mode location.

\section{POSITIONAL WEIGHT MATRIX SET}

852 Position Weight Matrices (PWMs) for TFs in Arabidopsis thaliana downloaded from

853 TRANSFAC (Wingender, 2008), JASPAR (Bryne et al., 2008), AGRIS (Davuluri et al., 2003),

854 and CIS-BP (Weirauch et al., 2014) databases. We developed a software program in Python in

855 order to compute the element-wise distance between PWM pairs; pairs with a distance less than

856 or equal to our empirical determined threshold of 0.9 were determined to be redundant. 
858 TSS peaks identified as described above in root and shoot were collected (approximately 50,000

859 peaks) and $6 \mathrm{~kb}$ sequences were extracted (TSS - $3 \mathrm{~kb}$, TSS $+3 \mathrm{~kb}$, centered at each TSS mode)

860 from the TAIR10 reference genome. As with the TSS-prediction models described earlier in the

861 methods, the TFBS Scanner suite (Morton and Megraw, 2014; Morton et al., 2014) was used to

862 scan each PWM over the extracted sequences, computing log-likelihood scores over these

863 regions. Regions of enrichment (ROEs) were defined on both the forward and reverse strands by

864 identifying the highest scoring region (the region with the largest sum of positive log-likelihood

865 scores) for each PWM across all promoter examples (Morton et al., 2014). PWMs with

866 cumulative log-likelihood score peaks up to $1 \mathrm{~kb}$ from the TSS mode were considered as regions

867 of enrichment for that PWM. The ROEs for each PWM were computed using an updated version

868 of ROEFinder software written in R. Our TEP-ROE model construction process is detailed in

869 Supplementary Figure 19.

\section{$870 \quad$ PROMOTER TILING}

871 Using TSS peaks identified as described in previous sections, $6 \mathrm{~kb}$ sequences (TSS - $3 \mathrm{~kb}$, TSS +

$8723 \mathrm{~kb}$, centered at each TSS mode) were extracted from the TAIR10 reference genome. Sequences

873 located from $1000 \mathrm{bp}$ upstream of TSS mode up to $500 \mathrm{bp}$ downstream of the TSS mode were

874 divided into 100-nt-wide, non-overlapping tiles. The PWMs were then scanned over each tile to

875 compute cumulative TFBS log-likelihood scores and percent overlap with open chromatin region

876 in root and shoot tissues. Our TEP-Tiled model construction is detailed in Supplementary Figure

87720.

\section{FEATURE SCALING}

879 Open chromatin features, as described in the feature generation section, all share the same 0-1

880 range and are interpretable as an "openness proportion" without modification. TFBS features,

881 however, are computed as a sum of positive log-likelihood scores over all nucleotides in the

882 region, where each nucleotide is taken as the starting point of a potential TF binding site; the log-

883 likelihood score is computed at this site using (1) the PWM associated with the TFs binding

884 domain, and (2) a local background nucleotide distribution model. The maximum possible value

885 for a TFBS feature is region length multiplied by the maximum possible log-likelihood value

$886 \mathrm{PWM}_{\text {scoremax }}$ of any binding site (i.e. the score of the PWMs consensus sequence); this value 
887 represents a theoretical 'maximal' case in which every nucleotide in a region represents the

888 consensus sequence of the PWM. To normalize TFBS features such that each feature

889 conceptually approximates a proportion of the maximum binding affinity, each TFBS feature is

890 divided by its region length. This puts the mean TFBS feature value on the same order of

891 magnitude as the mean OC feature value and allows for comparison between feature regions of 892 unequal length.

893 Model Training and Testing using nanoCAGE-XL TSSs

894 We constructed two classes of TSSs for use in training and testing of the ROE and Tiled models.

895 The "root" class (class 0 ) consists of TSSs associated with transcripts that are strongly expressed

896 in roots as compared to shoot, and the "shoot" class (class 1) consists of TSSs associated with

897 transcripts that are strongly expressed in shoots as compared with roots. For our purposes, we

898 defined "strongly expressed" as having an RNA-Seq data $\log _{2}$ fold-change value greater than 3.

899 Additionally, TSSs present in both classes were screened to ensure that each TSS peak contained

900 at least 300 reads in the tissue of its class label and each TSS-associated transcript had an RNA-

901 Seq expression value in both roots and shoots of at least 30 TPM. This ensured that both values

902 used for fold-change comparisons were reliably above background noise (Zavolan, 2015). The

903 remaining TSS peaks were then randomly partitioned into $80 \%$ training and 20\% independent

904 held-out test sets. Each data set contains balanced number of labeled classes. The Python Scikit-

905 learn library (Pedregosa et al., 2011) was used to implement L1-regularized logistic regression.

906 L1-model weights were tuned on the training set with 5-fold cross-validation (Supplementary

907 Figure 8), during which a range of parameter values was examined on the test partition. The

908 average of parameter values resulting in the best performance across the folds was selected for

909 final testing on the independent held-out test set (2.67 for TEP-ROE and 2.29 for TEP-Tiled).

910 All auROC and auPRC values are reported on the independent held-out test set for each model.

\section{Tissue of Expression Modeling Analyses}

912 Model Stability Assessment

913 In order to evaluate the stability of our TEP models, we performed two types of assessments.

914 First, we re-ran our TEP models 30 times on training and test sets with randomized 80/20

915 partitioning. For our second assessment, we removed the top N PWMs from our feature list $(\mathrm{n}=$

$9165,25,45$, etc). We used the new feature sets to re-train and test the model. The results from both 37 
917 of these assessments can be found in Supplementary Figures 10, 11, 12 and 13.

918 Comparison to model using TAIR10 annotated TSSs

919 To investigate the importance of using precise, experimentally-obtained TSS locations in

920 modeling, we generated a feature set as described above in the Feature Generation section of the

921 Methods using only annotated TAIR10 TSS locations. We then trained the TEP-ROE and TEP-

922 Tiled models on these annotated TSSs on nanoCAGE-XL data, as described in the Model

923 Training and Testing Methods section above.

924 Hard-coded promoter analyses

925 For our final TEP models constructed using nanoCAGE-XL data (TEP-ROE and TEP-Tiled,

926 including all feature types), promoter examples which were correctly classified with a high

927 probability $(\geq 0.9)$ were selected for our "hard-coded promoter" analysis. The sets of TFBS and

928 chromatin feature values for each of these promoters were extracted, and for each set the

929 following formula was applied:

930

$$
\begin{aligned}
& {\text { TFBS_SOP } \text { promoter }_{i}}=\sum_{j=1}^{\text {no_of_tfbs_features }} T F B S_{-} \text {score } e_{j} \cdot W_{j} \\
& O C_{-} S O P_{\text {promoter }_{i}}=\sum_{k=1}^{\text {no_of_oc_features }} O C_{-} \text {openness } s_{k} \cdot W_{k}
\end{aligned}
$$

$932 W$ is the model weight vector for each feature after training, $j$ and $k$ are the number of TFBS

933 features and OC features, respectively, and $i$ is the number of promoters. TFBS_SOP and

934 OC_SOP are the sum of products for TFBS features and OC features, respectively. The

935 distribution of the sum of products was computed for TFBS features and for chromatin features,

936 and the $5 \%$ tails of these distributions were considered for detecting putative hard-coded

937 promoters (Supplementary Figure 21). Promoters for which TFBS_SOP fell above the 95th

938 percentile and OC_SOP fell below the 5th percentile were labeled as putatively hard-coded.

939 Additionally, we extracted the features with the largest products for each of the TSSs for further

940 investigation. GO-enrichment analysis compared to the entire genome was performed for the

941 genes with putatively hard-coded promoters using GOATOOLS (Klopfenstein et al., 2018). 
943 The output of the classifier function for our trained L1-regularized logistic regression models is a

944 probability between zero and one, which represents the predicted likelihood of differential

945 expression in the two tissue types. Probabilities greater than 0.5 are labeled as "class 1 " or

946 "shoot", and probabilities less than 0.5 are labeled as "class 0" or "root". Logistic regression is a

947 Generalized Linear Model, where the probability of belonging to class 1 is a function of sum of

948 products of feature weights by feature values as follows:

$$
P_{i}=f\left(\sum_{j=1}^{\# \text { features }} W_{j} F_{i j}\right)=\frac{e^{\sum_{j=1}^{\# \text { features }} W_{j} F_{i j}}}{1+e^{\sum_{j=1}^{\# \text { features }} W_{j} F_{i j}}}
$$

950 where $P i$ is the probability that Promoter $i$ belongs to class 1 (shoot), $\mathrm{W} j$ is model weight, and

$951 \quad F i j$ is the $j$ th feature value for promoter $i$. Negative values of the feature product sum yield $\mathrm{P}_{i}$

952 (shoot) $<0.5$ (a root classification), and positive values of this feature product sum yield $\mathrm{P}_{i}$

953 (shoot) $>0.5$ (a shoot classification). Our in silico knockout process "zeroes out" selected feature

954 values and then computes the new model-predicted probability for a promoter. In the "single-

955 knockout" experiment, only one feature was removed from the equation at a time, in order to

956 determine effect on probability outcome for every promoter. Supplementary Tables 13 and 14

957 report cases with the largest probability 'shifts' across the 0.5 decision boundary, indicating

958 predicted high-probability 'flips' in tissue of greater expression upon in silico knockout of a

959 single TF-feature region.

960 Data and Model Availability

961 The full dataset of mapped, annotated nanoCAGE-XL TSS peaks, DNase I SIM peaks, and

962 RNA-Seq expression levels for root and shoot samples in our study is available on GBrowse at

963 http://megraw.cgrb.oregonstate.edu/suppmats/TissueOfExpressionPredictionDatasets. All raw

964 datasets, processed datasets, and model coefficient files are also made available for download.

965 Model training and evaluation pipelines are available upon request; these are designed to run on

966 a Sun Grid Engine computing cluster, and require user familiarity with the Unix/Linux operating

967 system, Make, Java, R, and Python; the pipelines cannot be supported by the authors on other

968 hardware systems. 
bioRxiv preprint doi: https://doi.org/10.1101/2020.09.01.278424; this version posted September 10, 2020. The copyright holder for this preprint (which was not certified by peer review) is the author/funder. All rights reserved. No reuse allowed without permission.

\section{Accession Numbers}

970 All raw reads have been deposited in the National Center for Biotechnology Information

971 Sequence Read Archive repository under the following accession numbers: OC-Seq (DNase I

972 SIM) - PRJNA285928; TSS-Seq (nanoCAGE-XL) - PRJNA658605; RNA-Seq -

973 PRJNA658596.

974 


\section{Supplementary Data}

976 The following materials are available in the online version of this article.

977 Supplementary Figure 1: TSS-Seq root and shoot sampled read depth saturation analysis

978 Supplementary Figure 2: Chromatin accessibility surrounding shared TSS mode in root

979 and shoot

980 Supplementary Figure 3: Proportion of top features shared by 3PEAT root and shoot

981 models

982 Supplementary Figure 4: False positive and false negative rates for 3PEAT root and shoot

983 models

984 Supplementary Figure 5: EMSA evaluations of putatively functional binding sites

985 Supplementary Figure 6: TEP-ROE model performance

986 Supplementary Figure 7: TEP-Tiled model performance

987 Supplementary Figure 8: Cross-validation ROC curves for ROE and Tiled models

988 Supplementary Figure 9: TEP model performance comparison

989 Supplementary Figure 10: Feature rank variability TEP-ROE model

990 Supplementary Figure 11: Feature rank variability TEP-Tiled model

991 Supplementary Figure 12: Feature removal performance plot for TEP-ROE model

992 Supplementary Figure 13: Feature removal performance plot for TEP-Tiled model

993 Supplementary Figure 14: Top-weighted feature comparison between TEP-Tiled and

994 enhancer model

995 Supplementary Figure 15: TEP-ROE rank correlation plot

996 Supplementary Figure 16: TEP-Tiled rank correlation plot

997 Supplementary Figure 17: ROE feature products vs openness

998 Supplementary Figure 18: Tiled feature products vs openness

999 Supplementary Figure 19: TEP-ROE model construction

1000 Supplementary Figure 20: TEP-Tiled model construction

1001 Supplementary Figure 21: Feature product sums for hard-codedness evaluation

1002 Supplementary Table 1: Number of TSS peaks and their mapped locations

1003 Supplementary Table 2: Number of TSS peaks and covered number of transcripts

1004 Supplementary Table 3: Basic statistics on TSS-seq, RNA-seq and OC data

1005 Supplementary Table 4: Basic RNA-seq expression statistics 
1006 Supplementary Table 5: Top weighted features for 3PEAT root and shoot models

1007 Supplementary Table 6: Cross-tissue model performance

1008 Supplementary Table 7: Cross-tissue 3PEAT-style GO-analysis for misclassified TSSs

1009 Supplementary Table 8: TEP-Tiled vs TEP-ROE models top feature comparison

1010 Supplementary Table 9: Top 30 features of enhancer-covering Tiled model

1011 Supplementary Table 10: Putatively "hardcoded" promoter examples from TEP-ROE

1012 Supplementary Table 11: Putatively "hardcoded" promoter examples from TEP-Tiled

1013 Supplementary Table 12: GO enrichment analyses for "hardcoded" promoter examples

1014 Supplementary Table 13: “In-silico knockout” results for TEP-ROE model

1015 Supplementary Table 14: “In-silico knockout" results for TEP-Tiled model

1016 Supplemental Data Set 1: (3PEAT_Model_weights.xlsx) 3PEAT logistic regression

1017 coefficients for 3PEAT root and shoot TSS location prediction models

1018 Supplemental Data Set 2: (TEP_Model_weights.xlsx) TEP logistic regression coefficients

1019 for ROE and Tiled Tissue-of-Expression-Prediction models

1020

1021 Acknowledgements

1022 Data generation for the study was supported by an NIH K99-R00 Pathway to Independence

1023 Award GM097188 to M.M. Algorithm design and computational analysis for the study was

1024 supported by an NSF CAREER Award 1750698 to M.M.

1025 Author Contributions

1026 MM designed the study. MI and SF carried out laboratory experiments for TSS-Seq, OC-Seq, 1027 and RNA-Seq dataset generation. MA performed algorithm implementation. MA and VF 1028 contributed to the design of data analysis experiments and to the evaluation of results. MA and 1029 VF worked together to select EMSA assay sites for examination, and VF carried out EMSA 1030 assays aided by OO. VF, RG, ZB, and SO contributed portions of the data analysis. OO and ZB 1031 contributed to data preparation for public distribution. MM, MA, and VF wrote the manuscript, 1032 all authors contributed to editing of the manuscript and approved the final version of the 1033 manuscript. We thank Dr. Uwe Ohler for ideas and discussions with M.M that inspired the 1034 conception of this study. We thank Dr. Ashok Prasad for his idea to investigate the existence of 42 
1035 "hard-coded" promoters. We thank Dr. Jason Cumbie for his help in preliminary evaluation of

1036 dataset quality, Natalie Brewer for her help in the PWM literature search, and Jordan Holdaway

1037 and Teresa Tran for their help in tissue preparation for the study. 


\section{References}

Agarwal, V., and Shendure, J. (2020). Predicting mRNA Abundance Directly from Genomic Sequence Using Deep Convolutional Neural Networks. Cell Reports 31, 107663.

Andersson, R. (2015). Promoter or enhancer, what's the difference? Deconstruction of established distinctions and presentation of a unifying model. BioEssays 37, 314-323.

Boyle, A.P., Guinney, J., Crawford, G.E., and Furey, T.S. (2008). F-Seq: a feature density estimator for high-throughput sequence tags. Bioinformatics 24, 2537-2538.

Bryne, J.C., Valen, E., Tang, M.H., Marstrand, T., Winther, O., da Piedade, I., Krogh, A., Lenhard, B., and Sandelin, A. (2008). JASPAR, the open access database of transcription factor-binding profiles: new content and tools in the 2008 update. Nucleic Acids Res 36, D102-106.

Cai, Y.M., Kallam, K., Tidd, H., Gendarini, G., Salzman, A., and Patron, N.J. (2020). Rational design of minimal synthetic promoters for plants. Nucleic Acids Res.

Chereji, R.V., Eriksson, P.R., Ocampo, J., Prajapati, H.K., and Clark, D.J. (2019). Accessibility of promoter DNA is not the primary determinant of chromatin-mediated gene regulation. Genome research 29, 1985-1995.

Cumbie, J.S., Filichkin, S.A., and Megraw, M. (2015a). Improved DNase-seq protocol facilitates high resolution mapping of DNase I hypersensitive sites in roots in Arabidopsis thaliana. Plant Methods 11, 42.

Cumbie, J.S., Ivanchenko, M.G., and Megraw, M. (2015b). NanoCAGE-XL and CapFilter: an approach to genome wide identification of high confidence transcription start sites. BMC Genomics 16, 597.

Davuluri, R.V., Sun, H., Palaniswamy, S.K., Matthews, N., Molina, C., Kurtz, M., and Grotewold, E. (2003). AGRIS: Arabidopsis gene regulatory information server, an information resource of Arabidopsis cis-regulatory elements and transcription factors. BMC Bioinformatics 4, 25.

Dong, X., Greven, M.C., Kundaje, A., Djebali, S., Brown, J.B., Cheng, C., Gingeras, T.R., Gerstein, M., Guigó, R., Birney, E., and Weng, Z. (2012). Modeling gene expression using chromatin features in various cellular contexts. Genome Biology 13, R53.

Feuerborn, A., and Cook, P.R. (2015). Why the activity of a gene depends on its neighbors. Trends in Genetics 31, 483-490.

Filichkin, S.A., and Megraw, M. DNase I SIM: A Simplified In-Nucleus Method for DNase I Hypersensitive Site Sequencing.

Forrest, A.R.R., Kawaji, H., Rehli, M., Kenneth Baillie, J., de Hoon, M.J.L., Haberle, V., Lassmann, T., Kulakovskiy, I.V., Lizio, M., Itoh, M., Andersson, R., Mungall, C.J., Meehan, T.F., Schmeier, S., Bertin, N., Jørgensen, M., Dimont, E., Arner, E., Schmidl, C., Schaefer, U., Medvedeva, Y.A., Plessy, C., Vitezic, M., Severin, J., Semple, C.A., Ishizu, Y., Young, R.S., Francescatto, M., Alam, I., Albanese, D., Altschuler, G.M., Arakawa, T., Archer, J.A.C., Arner, P., Babina, M., Rennie, S., Balwierz, P.J., Beckhouse, A.G., Pradhan-Bhatt, S., Blake, J.A., Blumenthal, A., Bodega, B., Bonetti, A., Briggs, J., Brombacher, F., Maxwell Burroughs, A., Califano, A., Cannistraci, C.V., Carbajo, D., Chen, Y., Chierici, M., Ciani, Y., Clevers, H.C., Dalla, E., Davis, C.A., Detmar, M., Diehl, A.D., Dohi, T., Drabløs, F., Edge, A.S.B., Edinger, M., Ekwall, K., Endoh, M., Enomoto, H., Fagiolini, M., Fairbairn, L., Fang, H., Farach-Carson, M.C., Faulkner, G.J., Favorov, A.V., 
Fisher, M.E., Frith, M.C., Fujita, R., Fukuda, S., Furlanello, C., Furuno, M., Furusawa, J.-i., Geijtenbeek, T.B., Gibson, A.P., Gingeras, T., Goldowitz, D., Gough, J., Guhl, S., Guler, R., Gustincich, S., Ha, T.J., Hamaguchi, M., Hara, M., Harbers, M., Harshbarger, J., Hasegawa, A., Hasegawa, Y., Hashimoto, T., Herlyn, M., Hitchens, K.J., Ho Sui, S.J., Hofmann, O.M., Hoof, I., Hori, F., Huminiecki, L., Iida, K., Ikawa, T., Jankovic, B.R., Jia, H., Joshi, A., Jurman, G., Kaczkowski, B., Kai, C., Kaida, K., Kaiho, A., Kajiyama, K., Kanamori-Katayama, M., Kasianov, A.S., Kasukawa, T., Katayama, S., Kato, S., Kawaguchi, S., Kawamoto, H., Kawamura, Y.I., Kawashima, T., Kempfle, J.S., Kenna, T.J., Kere, J., Khachigian, L.M., Kitamura, T., Peter Klinken, S., Knox, A.J., Kojima, M., Kojima, S., Kondo, N., Koseki, H., Koyasu, S., Krampitz, S., Kubosaki, A., Kwon, A.T., Laros, J.F.J., Lee, W., Lennartsson, A., Li, K., Lilje, B., Lipovich, L., Mackay-sim, A., Manabe, R.-i., Mar, J.C., Marchand, B., Mathelier, A., Mejhert, N., Meynert, A., Mizuno, Y., de Lima Morais, D.A., Morikawa, H., Morimoto, M., Moro, K., Motakis, E., Motohashi, H., Mummery, C.L., Murata, M., Nagao-Sato, S., Nakachi, Y., Nakahara, F., Nakamura, T., Nakamura, Y., Nakazato, K., van Nimwegen, E., Ninomiya, N., Nishiyori, H., Noma, S., Nozaki, T., Ogishima, S., Ohkura, N., Ohmiya, H., Ohno, H., Ohshima, M., Okada-Hatakeyama, M., Okazaki, Y., Orlando, V., Ovchinnikov, D.A., Pain, A., Passier, R., Patrikakis, M., Persson, H., Piazza, S., Prendergast, J.G.D., Rackham, O.J.L., Ramilowski, J.A., Rashid, M., Ravasi, T., Rizzu, P., Roncador, M., Roy, S., Rye, M.B., Saijyo, E., Sajantila, A., Saka, A., Sakaguchi, S., Sakai, M., Sato, H., Satoh, H., Savvi, S., Saxena, A., Schneider, C., Schultes, E.A., Schulze-Tanzil, G.G., Schwegmann, A., Sengstag, T., Sheng, G., Shimoji, H., Shimoni, Y., Shin, J.W., Simon, C., Sugiyama, D., Sugiyama, T., Suzuki, M., Suzuki, N., Swoboda, R.K., 't Hoen, P.A.C., Tagami, M., Takahashi, N., Takai, J., Tanaka, H., Tatsukawa, H., Tatum, Z., Thompson, M., Toyoda, H., Toyoda, T., Valen, E., van de Wetering, M., van den Berg, L.M., Verardo, R., Vijayan, D., Vorontsov, I.E., Wasserman, W.W., Watanabe, S., Wells, C.A., Winteringham, L.N., Wolvetang, E., Wood, E.J., Yamaguchi, Y., Yamamoto, M., Yoneda, M., Yonekura, Y., Yoshida, S., Zabierowski, S.E., Zhang, P.G., Zhao, X., Zucchelli, S., Summers, K.M., Suzuki, H., Daub, C.O., Kawai, J., Heutink, P., Hide, W., Freeman, T.C., Lenhard, B., Bajic, V.B., Taylor, M.S., Makeev, V.J., Sandelin, A., Hume, D.A., Carninci, P., Hayashizaki, Y., The, F.C., the, R.P., and Clst. (2014). A promoter-level mammalian expression atlas. Nature 507, 462-470.

Heintzman, N.D., Stuart, R.K., Hon, G., Fu, Y., Ching, C.W., Hawkins, R.D., Barrera, L.O., Van Calcar, S., Qu, C., Ching, K.A., Wang, W., Weng, Z., Green, R.D., Crawford, G.E., and Ren, B. (2007). Distinct and predictive chromatin signatures of transcriptional promoters and enhancers in the human genome. Nature Genetics 39, 311318.

Huminiecki, L., and Horbańczuk, J. (2017). Can We Predict Gene Expression by Understanding Proximal Promoter Architecture? Trends in Biotechnology 35, 530-546.

Ibrahim, M.M., Lacadie, S.A., and Ohler, U. (2015). JAMM: a peak finder for joint analysis of NGS replicates. Bioinformatics 31, 48-55.

Jaiswal, P., Avraham, S., Ilic, K., Kellogg, E.A., McCouch, S., Pujar, A., Reiser, L., Rhee, S.Y., Sachs, M.M., Schaeffer, M., Stein, L., Stevens, P., Vincent, L., Ware, D., and Zapata, F. (2005). Plant Ontology (PO): a Controlled Vocabulary of Plant Structures and 
Growth Stages. Comp Funct Genomics 6, 388-397.

Juven-Gershon, T., and Kadonaga, J.T. (2010). Regulation of gene expression via the core promoter and the basal transcriptional machinery. Dev Biol 339, 225-229.

Kadonaga, J.T. (2004). Regulation of RNA Polymerase II Transcription by Sequence-Specific DNA Binding Factors. Cell 116, 247-257.

Kadonaga, J.T. (2012). Perspectives on the RNA polymerase II core promoter. Wiley Interdisciplinary Reviews: Developmental Biology 1, 40-51.

Kim, T.-K., and Shiekhattar, R. (2015). Architectural and Functional Commonalities between Enhancers and Promoters. Cell 162, 948-959.

Klopfenstein, D.V., Zhang, L., Pedersen, B.S., Ramirez, F., Warwick Vesztrocy, A., Naldi, A., Mungall, C.J., Yunes, J.M., Botvinnik, O., Weigel, M., Dampier, W., Dessimoz, C., Flick, P., and Tang, H. (2018). GOATOOLS: A Python library for Gene Ontology analyses. Sci Rep 8, 10872.

Ko, J.Y., Oh, S., and Yoo, K.H. (2017). Functional Enhancers As Master Regulators of TissueSpecific Gene Regulation and Cancer Development. Molecules and cells 40, 169-177.

Kumari, S., and Ware, D. (2013). Genome-Wide Computational Prediction and Analysis of Core Promoter Elements across Plant Monocots and Dicots. PLoS ONE 8, e79011.

Lamesch, P., Berardini, T.Z., Li, D., Swarbreck, D., Wilks, C., Sasidharan, R., Muller, R., Dreher, K., Alexander, D.L., Garcia-Hernandez, M., Karthikeyan, A.S., Lee, C.H., Nelson, W.D., Ploetz, L., Singh, S., Wensel, A., and Huala, E. (2012). The Arabidopsis Information Resource (TAIR): improved gene annotation and new tools. Nucleic Acids Res 40, D1202-1210.

Langmead, B., and Salzberg, S.L. (2012). Fast gapped-read alignment with Bowtie 2. Nat Methods 9, 357-359.

Leng, N., Dawson, J.A., Thomson, J.A., Ruotti, V., Rissman, A.I., Smits, B.M., Haag, J.D., Gould, M.N., Stewart, R.M., and Kendziorski, C. (2013). EBSeq: an empirical Bayes hierarchical model for inference in RNA-seq experiments. Bioinformatics 29, 1035-1043.

Li, B., and Dewey, C.N. (2011). RSEM: accurate transcript quantification from RNA-Seq data with or without a reference genome. Bmc Bioinformatics 12.

Lu, Z., Marand, A.P., Ricci, W.A., Ethridge, C.L., Zhang, X., and Schmitz, R.J. (2019). The prevalence, evolution and chromatin signatures of plant regulatory elements. Nature Plants 5, 1250-1259.

Maher, K.A., Bajic, M., Kajala, K., Reynoso, M., Pauluzzi, G., West, D.A., Zumstein, K., Woodhouse, M., Bubb, K., Dorrity, M.W., Queitsch, C., Bailey-Serres, J., Sinha, N., Brady, S.M., and Deal, R.B. (2018). Profiling of Accessible Chromatin Regions across Multiple Plant Species and Cell Types Reveals Common Gene Regulatory Principles and New Control Modules. The Plant Cell 30, 15.

Megraw, M., Pereira, F., Jensen, S.T., Ohler, U., and Hatzigeorgiou, A.G. (2009). A transcription factor affinity-based code for mammalian transcription initiation. Genome Res 19, 644-656.

Mejía-Guerra, M.K., Li, W., Galeano, N.F., Vidal, M., Gray, J., Doseff, A.I., and Grotewold, E. (2015). Core Promoter Plasticity Between Maize Tissues and Genotypes Contrasts with Predominance of Sharp Transcription Initiation Sites. The Plant Cell 27, 3309.

Morton, T., and Megraw, M. (2014). 3PEAT TFBS-Scanner Toolset.

Morton, T., Petricka, J., Corcoran, D.L., Li, S., Winter, C.M., Carda, A., Benfey, P.N., 
Ohler, U., and Megraw, M. (2014). Paired-End Analysis of Transcription Start Sites in Arabidopsis Reveals Plant-Specific Promoter Signatures. The Plant Cell 26, 2746-2760.

Natarajan, A., Yardimci, G.G., Sheffield, N.C., Crawford, G.E., and Ohler, U. (2012). Predicting cell-type-specific gene expression from regions of open chromatin. Genome research 22, 1711-1722.

Ni, T., Corcoran, D., Rach, E., Song, S., Spana, E., Gao, Y., Ohler, U., and Zhu, J. (2010). A paired-end sequencing strategy to map the complex landscape of transcription initiation. Nature methods 7, 521-527.

Ong, C.-T., and Corces, V.G. (2011). Enhancer function: new insights into the regulation of tissue-specific gene expression. Nature Reviews Genetics 12, 283-293.

Pajoro, A., Madrigal, P., Muiño, J.M., Matus, J.T., Jin, J., Mecchia, M.A., Debernardi, J.M., Palatnik, J.F., Balazadeh, S., Arif, M., Ó’Maoiléidigh, D.S., Wellmer, F., Krajewski, P., Riechmann, J.-L., Angenent, G.C., and Kaufmann, K. (2014). Dynamics of chromatin accessibility and gene regulation by MADS-domain transcription factors in flower development. Genome Biology 15, R41.

Pedregosa, F., Varoquaux, G., Gramfort, A., Michel, V., Thirion, B., Grisel, O., Blondel, M., Prettenhofer, P., Weiss, R., Dubourg, V., Vanderplas, J., Passos, A., Cournapeau, D., Brucher, M., Perrot, M., and Duchesnay, E. (2011). Scikit-learn: Machine Learning in Python. J Mach Learn Res 12, 2825-2830.

Quinlan, A.R., and Hall, I.M. (2010). BEDTools: a flexible suite of utilities for comparing genomic features. Bioinformatics 26, 841-842.

Ricci, W.A., Lu, Z., Ji, L., Marand, A.P., Ethridge, C.L., Murphy, N.G., Noshay, J.M., Galli, M., Mejía-Guerra, M.K., Colomé-Tatché, M., Johannes, F., Rowley, M.J., Corces, V.G., Zhai, J., Scanlon, M.J., Buckler, E.S., Gallavotti, A., Springer, N.M., Schmitz, R.J., and Zhang, X. (2019). Widespread long-range cis-regulatory elements in the maize genome. Nature Plants 5, 1237-1249.

Rodgers-Melnick, E., Vera, D.L., Bass, H.W., and Buckler, E.S. (2016). Open chromatin reveals the functional maize genome. Proceedings of the National Academy of Sciences of the United States of America 113, E3177-E3184.

Ross, M.G., Russ, C., Costello, M., Hollinger, A., Lennon, N.J., Hegarty, R., Nusbaum, C., and Jaffe, D.B. (2013). Characterizing and measuring bias in sequence data. Genome Biology 14, R51.

Sandelin, A., Carninci, P., Lenhard, B., Ponjavic, J., Hayashizaki, Y., and Hume, D.A. (2007). Mammalian RNA polymerase II core promoters: insights from genome-wide studies. Nat Rev Genet 8, 424-436.

Sheffield, N.C., Thurman, R.E., Song, L., Safi, A., Stamatoyannopoulos, J.A., Lenhard, B., Crawford, G.E., and Furey, T.S. (2013). Patterns of regulatory activity across diverse human cell types predict tissue identity, transcription factor binding, and long-range interactions. Genome research 23, 777-788.

Smale, S.T., and Kadonaga, J.T. (2003). The RNA polymerase II core promoter. Annu Rev Biochem 72, 449-479.

Smith, A.M., Walsh, J.R., Long, J., Davis, C.B., Henstock, P., Hodge, M.R., Maciejewski, M., Mu, X.J., Ra, S., Zhao, S., Ziemek, D., and Fisher, C.K. (2020). Standard machine learning approaches outperform deep representation learning on phenotype prediction from transcriptomics data. BMC Bioinformatics 21, 119.

Snyder, Matthew W., Kircher, M., Hill, Andrew J., Daza, Riza M., and Shendure, J. (2016). 
Cell-free DNA Comprises an In Vivo Nucleosome Footprint that Informs Its Tissues-OfOrigin. Cell 164, 57-68.

Spitz, F., and Furlong, E.E.M. (2012). Transcription factors: from enhancer binding to developmental control. Nature Reviews Genetics 13, 613-626.

Staiger, D., Becker, F., Schell, J., Koncz, C., and Palme, K. (1991). Purification of tobacco nuclear proteins binding to a CACGTG motif of the chalcone synthase promoter by DNA affinity chromatography. Eur J Biochem 199, 519-527.

Sullivan, A.M., Arsovski, A.A., Lempe, J., Bubb, K.L., Weirauch, M.T., Sabo, P.J., Sandstrom, R., Thurman, R.E., Neph, S., Reynolds, A.P., Stergachis, A.B., Vernot, B., Johnson, A.K., Haugen, E., Sullivan, S.T., Thompson, A., Neri, F.V., 3rd, Weaver, M., Diegel, M., Mnaimneh, S., Yang, A., Hughes, T.R., Nemhauser, J.L., Queitsch, C., and Stamatoyannopoulos, J.A. (2014). Mapping and dynamics of regulatory DNA and transcription factor networks in A. thaliana. Cell Rep 8, 2015-2030.

Taher, L., Smith, R.P., Kim, M.J., Ahituv, N., and Ovcharenko, I. (2013). Sequence signatures extracted from proximal promoters can be used to predict distal enhancers. Genome biology 14, R117-R117.

Thomas, M.C., and Chiang, C.-M. (2006). The General Transcription Machinery and General Cofactors. Critical Reviews in Biochemistry and Molecular Biology 41, 105-178.

Vandenbon, A., and Nakai, K. (2010). Modeling tissue-specific structural patterns in human and mouse promoters. Nucleic acids research 38, 17-25.

Vera, D.L., Madzima, T.F., Labonne, J.D., Alam, M.P., Hoffman, G.G., Girimurugan, S.B., Zhang, J., McGinnis, K.M., Dennis, J.H., and Bass, H.W. (2014). Differential Nuclease Sensitivity Profiling of Chromatin Reveals Biochemical Footprints Coupled to Gene Expression and Functional DNA Elements in Maize. The Plant Cell 26, 3883.

Weirauch, Matthew T., Yang, A., Albu, M., Cote, A.G., Montenegro-Montero, A., Drewe, P., Najafabadi, Hamed S., Lambert, Samuel A., Mann, I., Cook, K., Zheng, H., Goity, A., van Bakel, H., Lozano, J.-C., Galli, M., Lewsey, M.G., Huang, E., Mukherjee, T., Chen, X., Reece-Hoyes, John S., Govindarajan, S., Shaulsky, G., Walhout, Albertha J.M., Bouget, F.-Y., Ratsch, G., Larrondo, Luis F., Ecker, Joseph R., and Hughes, Timothy R. (2014). Determination and Inference of Eukaryotic Transcription Factor Sequence Specificity. Cell 158, 1431-1443.

Wilken, M.S., Brzezinski, J.A., La Torre, A., Siebenthall, K., Thurman, R., Sabo, P., Sandstrom, R.S., Vierstra, J., Canfield, T.K., Hansen, R.S., Bender, M.A., Stamatoyannopoulos, J., and Reh, T.A. (2015). DNase I hypersensitivity analysis of the mouse brain and retina identifies region-specific regulatory elements. Epigenetics \& Chromatin 8, 8 .

Wingender, E. (2008). The TRANSFAC project as an example of framework technology that supports the analysis of genomic regulation. Briefings in Bioinformatics 9, 326-332.

Xi, H., Shulha, H.P., Lin, J.M., Vales, T.R., Fu, Y., Bodine, D.M., McKay, R.D.G., Chenoweth, J.G., Tesar, P.J., Furey, T.S., Ren, B., Weng, Z., and Crawford, G.E. (2007). Identification and characterization of cell type-specific and ubiquitous chromatin regulatory structures in the human genome. PLoS genetics 3, e136-e136.

Zavolan, M. (2015). Inferring gene expression regulatory networks from high-throughput measurements. Methods 85, 1-2.

Zhang, W., Zhang, T., Wu, Y., and Jiang, J. (2012a). Genome-wide identification of regulatory DNA elements and protein-binding footprints using signatures of open 
chromatin in Arabidopsis. Plant Cell 24, 2719-2731.

Zhang, W., Wu, Y., Schnable, J.C., Zeng, Z., Freeling, M., Crawford, G.E., and Jiang, J. (2012b). High-resolution mapping of open chromatin in the rice genome. Genome Res 22, 151-162. 